Submitted to Bernoulli

\title{
On uniform consistency of nonparametric tests I
}

\author{
MIKHAIL ERMAKOV \\ Institute of Problems of Mechanical Engineering RAS, Bolshoy pr., 61, VO, 1991178 St. \\ Petersburg and St. Petersburg State University, Universitetsky pr., 28, Petrodvoretz, 198504 \\ St. Petersburg, RUSSIA \\ E-mail: erm2512@gmail.com
}

For widespread nonparametric tests we point out necessary and sufficient conditions of uniform consistency of nonparametric sets of alternatives approaching to hypothesis. Nonparametric sets of alternatives can be defined both in terms of distribution functions and in terms of densities (or signals in the problem of signal detection in Gaussian white noise). In this part of paper such conditions are provided for $\chi^{2}$-tests having increasing number of cells with growth of sample size, Cramer-von Mises tests, tests generated $\mathbb{L}_{2}$ - norms of kernel estimators and tests generated quadratic forms of estimators of Fourier coefficients.

AMS 2000 subject classifications: Primary 62F03, 62G10, 62G20

Keywords: Cramer-von Mises tests, chi-squared test, consistency, goodness of fit tests, signal detection.

\section{Introduction}

Let $X_{1}, \ldots, X_{n}$ be sample of i.i.d.r.v.'s having c.d.f. $F \in \Im$. Here $\Im$ is set of all distribution functions of random variables having values into interval $(0,1)$

We explore problem of testing hypothesis

$$
\mathbb{H}_{0}: F(x)=F_{0}(x)=x, \quad x \in[0,1]
$$

versus sets of alternatives defined in terms of

distribution functions

$$
\mathbb{H}_{n}: F \in \Upsilon_{n}, \quad \Upsilon_{n} \subset \Im
$$

or in terms of densities $p(x)=1+f(x)=\frac{d F(x)}{d x}$

$$
\mathbb{H}_{1 n}: f \in \Psi_{n}, \quad \Psi_{n} \subset \mathbb{L}_{2}(0,1) .
$$

*The research has been supported by RFFI Grant 20-01-00273. 
Here $\mathbb{L}_{2}(0,1)$ is Hilbert space of all quadratically integrable functions $g(t), t \in(0,1)$ with $\mathbb{L}_{2}$-norm $\|g\|=\left(\int g^{2}(t) d t\right)^{1 / 2}$.

For part of setups the problem of goodness of fit testing for distribution function or density is replaced with the problem of signal detection in Gaussian white noise. This allows to simplify technical part of paper.

We are interested in uniform consistency of nonparametric tests. If test or test statistic is uniformly consistent for sets of alternatives, we say that these sets of alternatives are uniformly consistent for these tests or test statistics.

For setups mentioned above we point out necessary and sufficient conditions of uniform consistency of sets of alternatives (1.2) and (1.3) for test statistics of

Kolmogorov tests;

Cramer-von Mises tests;

chi-squared tests having increasing number of cells with growth of sample size;

tests generated quadratic forms of estimators of Fourier coefficients of orthogonal expansion of signal;

tests generated $\mathbb{L}_{2}$-norms of kernel estimators.

Last four of above mentioned tests statistics have quadratic structure. The results and proofs for these test statistics are similar. We provide these results in first part of paper. The results about Kolmogorov tests are provided in second part of paper.

Denote $\hat{F}_{n}$ - empirical distribution function of $X_{1}, \ldots, X_{n}$.

If sets of alternatives are defined in terms of distribution functions, necessary and sufficient conditions of consistency will be provided in the framework of distance method.

Test statistics can be considered as functionals $T_{n}\left(\hat{F}_{n}\right)$ depending on empirical distribution functions. Functionals $T_{n}(F)$ admits interpretation as norms or seminorms defined on the set of differences of distribution functions. Established uniform consistency of tests statistics on sets of alternatives

$$
\Upsilon_{n}\left(T_{n}, \rho_{n}\right)=\left\{F: T_{n}(F)>\rho_{n}>0, F \in \Im\right\}
$$

allows to make a conclusion about uniform consistency of any sequence of sets of alternatives $\Upsilon_{n}$ in terms of their distances or semidistances

$$
\inf _{F \in \Upsilon_{n}} T_{n}(F)
$$

from hypothesis.

For specially selected sequences $\rho_{n}, \rho_{n} \rightarrow 0$ as $n \rightarrow \infty$, in papers $[7,9,8]$ (see Theorems $6.3,4.3,5.2$ as well) we established uniform consistency of sets $\Upsilon_{n}\left(T_{n}, \rho_{n}\right)$ of alternatives for $\chi^{2}$-tests having increasing number of cells with growth of sample size, tests generated $\mathbb{L}_{2^{-}}$norms of kernel estimators and tests generated quadratic forms of estimators of Fourier coefficients Moreover asymptotic minimaxity of tests on these sets has been established. In this part of paper we establish uniform consistency of sets $\Upsilon_{n}\left(T, \rho_{n}\right)$ of alternatives for Cramer - von Mises test (see Theorem 7.1. Some similar results will be established for Kolmogorov test in the second part of paper.

imsart-bj ver. 2014/10/16 file: nuconxi.tex date: September 1, 2020 
Proof of results on uniform consistency of sets of alternatives (1.3) defined in terms of densities or signals are based on these results.

Problem of signal detection is considered for the following setup. We observe a realization of random process $Y_{n}(t)$ defined stochastic differential equation

$$
d Y_{n}(t)=f(t) d t+\frac{\sigma}{\sqrt{n}} d w(t), \quad t \in[0,1], \quad \sigma>0
$$

where $f \in \mathbb{L}_{2}(0,1)$ is unknown signal and $d w(t)$ is Gaussian white noise.

The following nonparametric sets of alternatives (see $[12,6,9,15,16,17,21,25]$ ) are often explored

$$
\mathbb{H}_{n}: f \in V_{n}=\left\{f:\|f\|^{2} \geq \rho_{n}, f \in U \subset \mathbb{L}_{2}(0,1)\right\}
$$

where $\rho_{n} \rightarrow 0$ as $n \rightarrow \infty$. Here $U$ is a convex set.

We answer on four questions given bellow. The answer on the first question is provided for problem of signal detection in Gaussian white boise and does not touch test statistics mentioned above.

For which bounded convex sets $U$ there are $\rho_{n} \rightarrow 0$ as $n \rightarrow \infty$ such that there is uniformly consistent sequence of tests for sets $V_{n}$ of alternatives?

We show that uniformly consistent test exists, if and only if, set $U$ is relatively compact (see Theorems 3.1 and 3.3). Note that necessary and sufficient condition of existence of consistent nonparametric estimator on nonparametric set is relative compactness of this set [14], [18]. The same compactness condition arises in solution of ill-posed inverse problems with deterministic errors [5]. The problem of existence of consistent tests has been explored for different setups. The most complete bibliography one can find in [10].

The answer on the next three questions is provided for i.i.d.r.v.'s model in the case of Cramer-von Mises tests and chi-squared tests. Test statistics generated quadratic forms of estimators of Fourier coefficients or tests generated $\mathbb{L}_{2^{-}}$norms of kernel estimators are explored for problem of signal detection in Gaussian white noise.

Let $\rho_{n}=n^{-r}, 0<r \leq 1 / 2$, and $r$ is fixed. How to define biggest bounded sets $U$ such that sets $V_{n}$ are uniformly consistent for one of above mentioned test statistics ?

We call such sets $U$ - maxisets The exact definition of maxisets is provided in section 2 . For $0<r<1 / 2$, for test statistics having quadratic structure we show (see Theorems 4.4, $5.1,6.1,7.2)$, that maxisets are bodies in Besov spaces $\mathbb{B}_{2 \infty}^{s}\left(P_{0}\right), P_{0}>0$. Here $r=\frac{2 s}{1+4 s}$ for chi-squared test statistics, test statistics being $\mathbb{L}_{2^{-}}$norms of kernel estimator and test statistics being quadratic forms of estimators of Fourier coefficients of signal. For Cramer- von Mises tests we have $r=\frac{s}{2+2 s}$.

If $r=1 / 2$, we could not find sets satisfying all requirements of the definition of maxisets. However, we show that bounded convex sets of functions having a fixed finite number of nonzero Fourier coefficients satisfy similar requirements. In further statements of this section for $r=1 / 2$, and therefore in the corresponding theorems, the maxisets can be replaced with such sets.

imsart-bj ver. 2014/10/16 file: nuconxi.tex date: September 1, 2020 
Uniform consistency of chi-squared tests and Cramer-von Mises tests for above mentioned Besov bodies has been established Ingster [15].

For nonparametric estimation the notion of maxisets has been introduced Kerkyacharian and Picard [19]. Maxisets of nonparametric estimators have been comprehensively explored in [4], [20], [27] (see also references therein). For nonparametric hypothesis testing completely different definition of maxisets has been introduced Autin, Clausel, Freyermuth and Marteau [2].

Let each set $\Psi_{n}$ be bounded in $\mathbb{L}_{2}(0,1)$. Then Cramer- von Mises tests, chi-squared tests, tests generated $\mathbb{L}_{2}$-norms of kernel estimators and quadratic forms of estimators of Fourier coefficients of signal are uniformly consistent, if and only if, these sets $\Psi_{n}$ of alternatives does not contain inconsistent sequence of simple alternatives $f_{n} \in \Psi_{n}$. In other words sets of alternatives are uniformly consistent, if and only if, all sequences of simple alternatives $f_{n} \in \Psi_{n}$ are consistent. Thus the problem of uniform consistency for sets $\Psi_{n}$ of alternatives is reduced to the problem of consistency of any sequence of simple alternatives $f_{n} \in \Psi_{n}$.

How to describe all consistent and inconsistent sequences of simple alternatives having given rate of convergence to hypothesis ?

We explore this problem as problem of testing hypothesis

$$
\overline{\mathbb{H}}_{0}: f(x)=0, \quad x \in[0,1],
$$

versus sequence of simple alternatives

$$
\overline{\mathbb{H}}_{n}: f=f_{n}, \quad c n^{-r} \leq\left\|f_{n}\right\| \leq C n^{-r},
$$

where $0<r \leq 1 / 2$ and $0<c<C<\infty$.

For above mentioned test statistics answer on this question is provided in terms of concentration of Fourier coefficients (Theorems 4.1 and 4.2). In Theorem 4.5 we propose the following interpretation of these results:

sequence of simple alternatives $f_{n}, c n^{-r} \leq\left\|f_{n}\right\| \leq C n^{-r}$, is consistent, if and only if, functions $f_{n}$ admit representation as functions $f_{1 n}$ from maxiset with the same rate of convergence to hypothesis plus functions $f_{n}-f_{1 n}$ orthogonal to functions $f_{1 n}$.

In Theorem 4.6 we show that, for any $\varepsilon>0$, there are maxiset and functions $f_{1 n}$ from maxiset such that the differences of type II error probabilities for alternatives $f_{n}$ and $f_{1 n}$ is smaller $\varepsilon$ and $f_{1 n}$ is orthogonal to $f_{n}-f_{1 n}$.

Thus, each function of consistent sequence of alternatives with fixed rate of convergence to hypothesis contains sufficiently smooth function as an additive component and this function carries almost all information on its type II error probability.

What can we say about properties of consistent and inconsistent sequences of alternatives having fixed rate of convergence to hypothesis in $\mathbb{L}_{2}$ - norm?

In Theorem 4.7 we establish that asymptotic of type II error probabilities of sums of alternatives from consistent and inconsistent sequences coincides with the asymptotic for consistent sequence.

imsart-bj ver. 2014/10/16 file: nuconxi.tex date: September 1, 2020 
We call sequence of alternatives $f_{n}$ purely consistent if there does not exist inconsistent sequence of alternatives $f_{2 n}$ having the same rates of convergence to hypothesis and such that $f_{2 n}$ are orthogonal to $f_{n}-f_{2 n}$.

It is easy to show that any sequence of alternatives from maxisets with fixed rates of convergence to hypothesis is purely consistent.

In Theorem 4.8, in terms of concentration of Fourier coefficients we point out analytic assignment of purely consistent sequences of alternatives.

In Theorem 4.9 we show that, for any $\varepsilon>0$, for any purely consistent sequence of alternatives $f_{n}, c n^{-r} \leq\left\|f_{n}\right\| \leq C n^{-r}$, there are maxiset and some sequence $f_{1 n}$ from this maxiset, such that there holds $\left\|f_{n}-f_{1 n}\right\| \leq \varepsilon n^{-r}$.

Paper is organized as follows. In section 2 we introduce main definitions. In section 3 , the answer on the first question is provided. In sections $4,5,6$ and 7 , for $0<r<1 / 2$, above mentioned results are established respectively for test statistics based on quadratic forms of estimators of Fourier coefficients, $\mathbb{L}_{2}-$ norms of kernel estimators, $\chi^{2}$-tests and Cramer- von Mises tests. In section 8 we focus on the case $r=\frac{1}{2}$.

Proof of all Theorems is provided in Appendix.

We use letters $c$ and $C$ as a generic notation for positive constants. Denote $\mathbf{1}_{\{A\}}$ the indicator of an event $A$. Denote $[a]$ whole part of real number $a$. For any two sequences of positive real numbers $a_{n}$ and $b_{n}, a_{n} \asymp b_{n}$ implies $c<a_{n} / b_{n}<C$ for all $n$ and $a_{n}=o\left(b_{n}\right)$ implies $a_{n} / b_{n} \rightarrow 0$ as $n \rightarrow \infty$. For any complex number $z$ denote $\bar{z}$ complex conjugate number.

Denote

$$
\Phi(x)=\frac{1}{\sqrt{2 \pi}} \int_{-\infty}^{x} \exp \left\{-t^{2} / 2\right\} d t, \quad x \in \mathbb{R}^{1},
$$

standard normal distribution function.

Let $\phi_{j}, 1 \leq j<\infty$, be orthonormal system of functions in $\mathbb{L}_{2}(0,1)$. For each $P_{0}>0$ define set

$$
\overline{\mathbb{B}}_{2 \infty}^{s}\left(P_{0}\right)=\left\{f: f=\sum_{j=1}^{\infty} \theta_{j} \phi_{j}, \sup _{\lambda>0} \lambda^{2 s} \sum_{j>\lambda} \theta_{j}^{2} \leq P_{0}, \theta_{j} \in \mathbb{R}^{1}\right\} .
$$

If some assumptions about basis $\phi_{j}, 1 \leq j<\infty$, holds, functional space

$$
\overline{\mathbb{B}}_{2 \infty}^{s}=\left\{f: f=\sum_{j=1}^{\infty} \theta_{j} \phi_{j}, \sup _{\lambda>0} \lambda^{2 s} \sum_{j>\lambda} \theta_{j}^{2}<\infty, \theta_{j} \in \mathbb{R}^{1}\right\}
$$

is Besov space $\mathbb{B}_{2 \infty}^{s}$ (see [27]). In particular, $\overline{\mathbb{B}}_{2 \infty}^{s}$ is Besov space if $\phi_{j}, 1 \leq j<\infty$, is trygonometric basis.

$$
\begin{aligned}
& \text { If } \phi_{j}(t)=\exp \{2 \pi i j x\}, x \in(0,1), j=0, \pm 1, \ldots \text {, denote } \\
& \qquad \mathbb{B}_{2 \infty}^{s}\left(P_{0}\right)=\left\{f: f=\sum_{j=-\infty}^{\infty}, \theta_{j} \phi_{j}, \sup _{\lambda>0} \lambda^{2 s} \sum_{|j|>\lambda}\left|\theta_{j}\right|^{2} \leq P_{0}\right\} .
\end{aligned}
$$


Since here $\phi_{j}$ are complex functions, then $\theta_{j}$ are complex numbers as well and $\theta_{j}=\bar{\theta}_{-j}$ for all $-\infty<j<\infty$.

For the same basis denote

$$
\tilde{\mathbb{B}}_{2 \infty}^{s}\left(P_{0}\right)=\left\{f: f=\sum_{j=-\infty}^{\infty} \theta_{j} \phi_{j}, f \in \mathbb{B}_{2 \infty}^{s}\left(P_{0}\right), \theta_{0}=0\right\} .
$$

Balls in Nikolskii classes

$$
\int\left(f^{(l)}(x+t)-f^{(l)}(x)\right)^{2} d x \leq L|t|^{2(s-l)}, \quad\|f\|<C,
$$

are Besov balls in $\mathbb{B}_{2 \infty}^{s}$. Here $l=[s]$.

\section{Main definitions}

\subsection{Consistency and $n^{-r}$-consistency}

For any test $K_{n}$ denote $\alpha\left(K_{n}\right)$ its type I error probability, and $\beta\left(K_{n}, f\right)$ its type II error probability for alternative $f \in \mathbb{L}_{2}(0,1)$. Similar notation $\beta\left(K_{n}, F\right)$ is implemented if alternative is c.d.f. $F$.

Definition of consistency will be slightly different in each section. In section 3 problem of existence of uniformly consistent tests and uniform consistency of sets of alternatives is considered among all tests.

In section 4 consistency is considered for a fixed sequence of test statistics $T_{n}$. For kernel-based tests and chi-squared tests, consistency is explored for whole population of test statistics depending on kernel width and number of cells respectively. In section 7 we have only one test statistic.

We showed that problem of uniform consistency of sets of alternatives is reduced to the problem of consistency of sequences of simple alternatives. Thus, in sections $4-7$, we explore this setup.

Below we provide definition of consistency for setup of sections 4 and 7. In sections 5 and 6 the definitions will be different in the sense mentioned above.

We say that sequence of simple alternatives $f_{n}$ is consistent if for any $\alpha, 0<\alpha<1$, for sequence of tests $K_{n}, \alpha\left(K_{n}\right)=\alpha(1+o(1))$, generated test statistics $T_{n}$, there holds

$$
\limsup _{n \rightarrow \infty} \beta\left(K_{n}, f_{n}\right)<1-\alpha .
$$

If $c n^{-r}<\left\|f_{n}\right\|<C n^{-r}$ additionally, we say that sequence of alternatives $f_{n}$ is $n^{-r}$ consistent (see [30]).

We say that sequence of alternatives $f_{n}$ is inconsistent if, for each sequence of tests $K_{n}$ generated test statistics $T_{n}$, there holds

$$
\liminf _{n \rightarrow \infty}\left(\alpha\left(K_{n}\right)+\beta\left(K_{n}, f_{n}\right)\right) \geq 1 .
$$

imsart-bj ver. 2014/10/16 file: nuconxi.tex date: September 1, 2020 
Suppose we consider problem of testing hypothesis (1.1) versus alternatives (1.3) where $\Psi_{n}$ can be also sets of signals.

For tests $K_{n}, \alpha\left(K_{n}\right)=\alpha+o(1), 0<\alpha<1$, generated test statistics $T_{n}$ denote $\beta\left(K_{n}, \Psi_{n}\right)=\sup _{f \in \Psi_{n}} \beta\left(K_{n}, f\right)$. We say that sequence of sets $\Psi_{n}$ of alternatives is uniformly consistent if

$$
\limsup _{n \rightarrow \infty} \beta\left(K_{n}, \Psi_{n}\right)<1-\alpha
$$

For sets of alternatives $\Upsilon_{n}$ defined (1.2) definition of uniform consistency is the same.

\subsection{Purely consistent sequences}

We say that $n^{-r}$ - consistent sequence of alternatives $f_{n}$ is purely $n^{-r}$-consistent if there does not exist subsequence $f_{n_{i}}$ such that $f_{n_{i}}=f_{1 n_{i}}+f_{2 n_{i}}$ where $f_{2 n_{i}}$ is orthogonal to $f_{1 n_{i}}$ and sequence $f_{2 n_{i}},\left\|f_{2 n_{i}}\right\|>c_{1} n^{-r}$, is inconsistent.

\subsection{Maxisets}

Let $\phi_{j}, 1 \leq j<\infty$, be orthonormal basis in $\mathbb{L}_{2}(0,1)$. We say that a set $U, U \subset$ $\mathbb{L}_{2}(0,1)$, is ortho-symmetric with respect to this basis if $f=\sum_{j=1}^{\infty} \theta_{j} \phi_{j} \in U$ implies $\tilde{f}=\sum_{j=1}^{\infty} \tilde{\theta}_{j} \phi_{j} \in U$ for any $\tilde{\theta}_{j}=\theta_{j}$ or $\tilde{\theta}_{j}=-\theta_{j}, j=1,2, \ldots$

For closed ortho -symmetric bounded convex set $U, U \subset \mathbb{L}_{2}(0,1)$, denote $\Xi$ functional space with unite ball $U$.

For the problem of signal detection we call bounded ortho-symmetric closed set $U$, $U \subset \mathbb{L}_{2}(0,1)$, maxiset and functional space $\Xi$ maxispace if

i. any subsequence of alternatives $f_{n_{i}} \in \gamma U, c n_{i}^{-r}<\left\|f_{n_{i}}\right\|<C n_{i}^{-r}, n_{i} \rightarrow \infty$ as $i \rightarrow \infty$, is consistent,

ii. if $f \notin \Xi$, then, in any convex, ortho-symmetric set $V$ that contains $f$, there is inconsistent subsequence of alternatives $f_{n_{i}} \in V, c n_{i}^{-r}<\left\|f_{n_{i}}\right\|<C n_{i}^{-r}$, where $n_{i} \rightarrow \infty$ as $i \rightarrow \infty$.

ii. implies that $U$ is the largest set satisfying $i$.

For problem of hypothesis testing on a density, in definition of maxiset we make additional assumption:

ii. is considered only for functions $f=1+\sum_{i=1}^{\infty} \theta_{i} \phi_{i}$ (or $f=1+\sum_{|i| \geq 1}^{\infty} \theta_{i} \phi_{i}$ ) satisfying the following condition.

D. There is $l_{0}=l_{0}(f)$ such that, for all $l>l_{0}$, functions $1+\sum_{|i|>l}^{\infty} \theta_{i} \phi_{j}$ are nonnegative (are densities). ii.

D allows to analyze tails $f_{n_{j}}=\sum_{|i| \geq j} \theta_{i} \phi_{i}$ of orthogonal expansions of $f$ to establish

It is clear that if, $U$ is maxiset, then $\gamma U, 0<\gamma<\infty$, is maxiset as well. 
Simultaneous assumptions of convexity and ortho-symmetry of set $V$ is rather strong. If $f \in V, f=\sum_{i=1}^{\infty} \theta_{i} \phi_{i}$, then any $f_{\eta} \in V$ with $f_{\eta}=\sum_{i=1}^{\infty} \eta_{i} \phi_{i},\left|\eta_{i}\right|<\left|\theta_{i}\right|, 1 \leq i<\infty$.

Test statistics of tests generated $\mathbb{L}_{2}$ - norms of kernel estimators and Cramer-von Mises tests admit representation as a linear combination of squares of estimators of Fourier coefficients. Therefore, for these test statistics, consistency of sequence $f_{n}$ implies consistency of any sequence of ortho-symmetric functions $\tilde{f}_{n}$ generated $f_{n}$. Moreover, type II error probabilities of sequences $f_{n}$ and $\tilde{f}_{n}$ have the same asymptotic. Thus the requirement of ortho-symmetry seems natural for test statistics admitting representation as a liner combination of squares of estimators of Fourier coefficients. For chi-squared tests, by Theorem 6.1 given in what follows, similar situation takes place.

\subsection{Another approach to definition of maxisets}

Requirement of ortho-symmetry of set $U$ does not allow to call maxiset any convex set $W$ generated equivalent norm in $\Xi$. In definition of maxiset given below we do not make such an assumption.

Let $\Xi \subset \mathbb{L}_{2}(0,1)$ be Banach space with a norm $\|\cdot\|_{\Xi}$. Denote $U=\left\{f:\|f\|_{\Xi} \leq \gamma, f \in\right.$ $\Xi\}, \gamma>0$, a ball in $\Xi$.

Define subspaces $\Pi_{k}, 1 \leq k<\infty$, by induction.

Denote $d_{1}=\max \{\|f\|, f \in U\}$ and denote $e_{1}$ function $e_{1} \in U$ such that $\left\|e_{1}\right\|=d_{1}$. Denote $\Pi_{1}$ linear subspace generated vector $e_{1}$.

For $i=2,3, \ldots$ denote $d_{i}=\max \left\{\rho\left(f, \Pi_{i-1}\right), f \in U\right\}$ with $\rho\left(f, \Pi_{i-1}\right)=\min \{\| f-$ $\left.g \|, g \in \Pi_{i-1}\right\}$. Define function $e_{i}, e_{i} \in U$, such that $\rho\left(e_{i}, \Pi_{i-1}\right)=d_{i}$. Denote $\Pi_{i}$ linear subspace generated functions $e_{1}, \ldots, e_{i}$.

For any function $f \in \mathbb{L}_{2}(0,1)$ denote $f_{\Pi_{i}}$ projection of function $f$ on subspace $\Pi_{i}$ and denote $\tilde{f}_{i}=f-f_{\Pi_{i}}$.

Thus we associate with each $f \in \mathbb{L}_{2}(0,1)$ sequence of functions $\tilde{f}_{i}, \tilde{f}_{i} \rightarrow 0$ as $i \rightarrow \infty$.

For the problem of signal detection we say that set $U$ is maxiset for test statistics $T_{n}$ and $\Xi$ is maxispace if the following two statements take place.

i. any subsequence of alternatives $f_{n_{j}} \in U, c n_{j}^{-r}<\left\|f_{n_{j}}\right\|<C n_{j}^{-r}, n_{j} \rightarrow \infty$ as $j \rightarrow \infty$, is consistent,.

ii. for any $f \in \mathbb{L}_{2}(0,1), f \notin \Xi$, there are sequences $i_{n}$ and $j_{i_{n}}$ with $i_{n} \rightarrow \infty, j_{i_{n}} \rightarrow \infty$ as $n \rightarrow \infty$, such that subsequence $\tilde{f}_{i_{n}}$ is inconsistent and $c j_{i_{n}}^{-r}<\left\|\tilde{f}_{i_{n}}\right\|<C j_{i_{n}}^{-r}$.

For problem of hypothesis testing on a density, ii. is verified only for functions $f$ such that $1+\tilde{f}_{i}$ are densities for all $i>i_{0}$.

We provide proofs of Theorems for definition of maxisets in terms of subsection 2.3. However it is easy to see that slight modification of this reasoning provide proofs for definition of subsection 2.4 as well. Basis $\phi_{j}, 1 \leq j<\infty$, in subsection 2.3 coincides in this reasoning with basis $e_{j}$.

imsart-bj ver. 2014/10/16 file: nuconxi.tex date: September 1, 2020 


\section{Necessary and sufficient conditions of uniform consistency}

We consider problem of signal detection in Gaussian white noise discussed in Introduction. Problem is explored in terms of sequence model.

Stochastic differential equation (1.4) can be rewritten in terms of a sequence model based on orthonormal system of functions $\phi_{j}, 1 \leq j<\infty$, in the following form

$$
y_{j}=\theta_{j}+\frac{\sigma}{\sqrt{n}} \xi_{j}, \quad 1 \leq j<\infty
$$

where

$$
y_{j}=\int_{0}^{1} \phi_{j} d Y_{n}(t), \quad \xi_{j}=\int_{0}^{1} \phi_{j} d w(t) \quad \text { and } \quad \theta_{j}=\int_{0}^{1} f \phi_{j} d t .
$$

Denote $\boldsymbol{y}=\left\{y_{j}\right\}_{j=1}^{\infty}$ and $\boldsymbol{\theta}=\left\{\theta_{j}\right\}_{j=1}^{\infty}$.

We can consider $\boldsymbol{\theta}$ as a vector in Hilbert space $\mathbb{H}$ with the norm $\|\boldsymbol{\theta}\|=\left(\sum_{j=1}^{\infty} \theta_{j}^{2}\right)^{1 / 2}$. We implement the same notation $\|\cdot\|$ in $\mathbb{L}_{2}$ and in $\mathbb{H}$. Sense of this notation will be always clear from context.

In this notation the problem of hypothesis testing can be rewritten in the following form. One needs to test the hypothesis

$$
\mathbb{H}_{0}: \boldsymbol{\theta}=\mathbf{0}
$$

versus alternatives

$$
\mathbb{H}_{n}: \boldsymbol{\theta} \in V_{n}=\left\{\boldsymbol{\theta}:\|\boldsymbol{\theta}\| \geq \rho_{n}, \boldsymbol{\theta} \in U, U \subset \mathbb{H}\right\} .
$$

Here $U$ is bounded convex set.

We say that $\mathbf{0}=\{0,0, \ldots\}$ is inner point of set $U$ if for any $\boldsymbol{y} \in \mathbb{H}$ there is $\lambda>0$ such that $\lambda \boldsymbol{y} \in U$ and $-\lambda \boldsymbol{y} \in U$.

Theorem 3.1. Suppose that bounded set $U$ is convex and $\mathbf{0}$ is inner point of $U$. Then there is sequence $\rho_{n} \rightarrow 0$ as $n \rightarrow \infty$ such that there is uniformly consistent sequence of tests for sets of alternatives $V_{n}$ with this sequence $\rho_{n}$, if and only if, set $U$ is relatively compact.

If set $U$ is relatively compact, there is consistent estimator (see [14] and [18]). Therefore we can choose $\mathbb{L}_{2}$-norm of consistent estimator as uniformly consistent test statistics.

Remark 3.1. Suppose $K$ is convex hull of points $\boldsymbol{\theta}_{1}, \boldsymbol{\theta}_{2}, \ldots$ and $\mathbf{0}$ is inner point of $K$. Suppose $K$ is not relatively compact and $K \subset U$ where the set $U$ is not necessarily convex. Then, by Theorem 3.1, for problem of testing hypothesis (3.2) versus alternatives (3.3), there does not exist uniformly consistent tests for all sequences $\rho_{n} \rightarrow 0$ as $n \rightarrow \infty$. 
Version of Theorem 3.1 holds for problem of testing hypothesis on a density in a following setup. Let $\mathbf{P}$ be probability measure on $\sigma$-algebra $\Im$ defined on set $D$. Denote $\mathbb{L}_{2}(\mathbf{P})$ set of measurable functions $f: D \rightarrow \mathbb{R}^{1}$ such that

$$
\int_{S} f^{2} d \mathbf{P}<\infty .
$$

Let $X_{1}, \ldots, X_{n}$ be i.i.d.r.v.'s having probability measure $\mathbf{Q}$, having density $q=\frac{d \mathbf{Q}}{d \mathbf{P}}$ such that $q \in \mathbb{L}_{2}(\mathbf{P})$.

Problem is to test hypothesis $\mathbb{H}_{0}: q(s)=1$ for all $s \in D$ versus alternative $\mathbb{H}_{1}$ : $q(s)-1 \in V_{n}=\left\{f:\|f\| \geq \rho_{n}, f \in U, U \subset \mathbb{L}_{2}(\mathbf{P})\right\}$. Here $\|f\|$ denotes $\mathbb{L}_{2}(\mathbf{P})$ - norm of function $f$. and $U$ is bounded convex set in $\mathbb{L}_{2}(\mathbf{P})$

Define function $\mathbf{0}(s)=0$ for all $s \in D$.

Theorem 3.2. Suppose that set $U$ is bounded convex into $\mathbb{L}_{2}(\mathbf{P})$. Let set $U$ be such that for any function $f \in U$ function $1+f$ is probability density. Let $\mathbf{0}$ be inner point of $U$. Then there is sequence $\rho_{n} \rightarrow 0$ as $n \rightarrow \infty$ such that there is uniformly consistent sequence of tests for sets of alternatives $V_{n}$ with this sequence $\rho_{n}$, if and only if, set $U$ is relatively compact.

Reasoning in the proof of Theorem 3.2 coincides with the reasoning of proof of Theorem 3.1 with unique difference we implement Theorem 4.1 in [10] instead of Theorem 5.3 in [10]. We omit this reasoning.

Similar Theorem holds for problem of signal detection in linear inverse ill-posed problem.

In Hilbert space $\mathbb{H}$, we observe a realization of Gaussian random vector

$$
\boldsymbol{y}=A \boldsymbol{\theta}+\epsilon \boldsymbol{\xi}, \quad \epsilon>0
$$

where $A: \mathbb{H} \rightarrow \mathbb{H}$ is known linear operator and $\boldsymbol{\xi}$ is Gaussian random vector having known covariance operator $R: \mathbb{H} \rightarrow \mathbb{H}$ and $\mathbf{E}[\boldsymbol{\xi}]=0$.

We explore the same problem of hypothesis testing $\mathbb{H}_{0}: \boldsymbol{\theta}=0$ versus alternatives $\mathbb{H}_{n}: \boldsymbol{\theta} \in V_{n}$.

For any operator $S: \mathbb{H} \rightarrow \mathbb{H}$ denote $\mathfrak{R}(S)$ the rangespace of $S$.

Suppose that the nullspaces of $A$ and $R$ equal zero and $\mathfrak{R}(A) \subseteq \mathfrak{R}\left(R^{1 / 2}\right)$.

Theorem 3.3. Let operator $R^{-1 / 2} A$ be bounded. Suppose that bounded set $U$ is convex and $\mathbf{0}$ is inner point of $U$. Then the statement of Theorem 3.1 holds.

Remark 3.2. In papers another definition of uniform consistency is often explored (see, for example, [15]). In this definition, (2.3) is replaced with the requirement of existence of sequence of tests $K_{n}$ such that $\alpha\left(K_{n}\right) \rightarrow 0$ and $\beta\left(K_{n}, V_{n}\right) \rightarrow 0$ as $n \rightarrow \infty$. By Theorem on exponential decay of type $I$ and type II error probabilities (see [23] and [29]), the statements of Theorems 3.1 - 3.3 for this definition of consistency follows from these Theorems. 


\section{Quadratic test statistics}

\subsection{General setup}

We explore problem of signal detection in Gaussian white noise (1.4) and (1.7) with $0<r<1 / 2$ discussed in Introduction. Problem is provided in terms of sequence model (3.1).

If $U$ is compact ellipsoid

$$
U=\left\{\boldsymbol{\theta}: \sum_{j=1}^{\infty} a_{j} \theta_{j}^{2} \leq P_{0}, \boldsymbol{\theta}=\left\{\theta_{j}\right\}_{j=1}^{\infty}, \theta_{j} \in \mathbb{R}^{1}\right\}
$$

with $a_{j}>0, a_{j} \rightarrow \infty$ as $j \rightarrow \infty$, asymptotically minimax test statistics for sets of alternatives $V_{n}$ are quadratic forms

$$
T_{n}\left(Y_{n}\right)=\sum_{j=1}^{\infty} \kappa_{n j}^{2} y_{j}^{2}-\sigma^{2} n^{-1} \rho_{n}
$$

with some specially defined coefficients $\kappa_{n j}^{2}$ (see Ermakov [6]). Here $\rho_{n}=\sum_{j=1}^{\infty} \kappa_{n j}^{2}$.

If coefficients $\kappa_{n j}^{2}$ satisfy some regularity assumptions, test statistics $T_{n}\left(Y_{n}\right)$ are asymptotically minimax (see [9]) for wider sets of alternatives

$$
\mathbb{H}_{n}: f \in \Upsilon_{n}\left(R_{n}, c\right)=\left\{f: R_{n}(f)>c, f \in \mathbb{L}_{2}(0,1)\right\}
$$

with

$$
R_{n}(f)=A_{n}(\boldsymbol{\theta})=\sigma^{-4} n^{2} \sum_{j=1}^{\infty} \kappa_{n j}^{2} \theta_{j}^{2}
$$

and $f=\sum_{j=1}^{\infty} \theta_{j} \phi_{j}$.

A sequence of tests $L_{n}, \alpha\left(L_{n}\right)=\alpha(1+o(1)), 0<\alpha<1$, is called asymptotically minimax if, for any sequence of tests $K_{n}, \alpha\left(K_{n}\right) \leq \alpha$, there holds

$$
\liminf _{n \rightarrow \infty}\left(\beta\left(K_{n}, \Upsilon_{n}\left(R_{n}, c\right)\right)-\beta\left(L_{n}, \Upsilon_{n}\left(R_{n}, c\right)\right)\right) \geq 0 .
$$

Sequence of test statistics $T_{n}$ is called asymptotically minimax if tests generated test statistics $T_{n}$ are asymptotically minimax.

We make the following assumptions.

A1. For each $n$ sequence $\kappa_{n j}^{2}$ is decreasing.

A2. There are positive constants $C_{1}$ and $C_{2}$ such that, for each $n$, there holds

$$
C_{1}<A_{n}=\sigma^{-4} n^{2} \sum_{j=1}^{\infty} \kappa_{n j}^{4}<C_{2} .
$$

A3. There are positive constants $c_{1}$ and $c_{2}$ such that $c_{1} n^{-2 r} \leq \rho_{n} \leq c_{2} n^{-2 r}$. 
Denote $\kappa_{n}^{2}=\kappa_{n k_{n}}^{2}$ with $k_{n}=\sup \left\{k: \sum_{j<k} \kappa_{n j}^{2} \leq \frac{1}{2} \rho_{n}\right\}$.

A4. There are $C_{1}$ and $\lambda>1$ such that, for any $\delta>0$ and for each $n$ we have

$$
\kappa_{\left[n,(1+\delta) k_{n}\right]}^{2}<C_{1}(1+\delta)^{-\lambda} \kappa_{n}^{2}
$$

A5. There holds $\kappa_{n 1}^{2} \asymp \kappa_{n}^{2}$ as $n \rightarrow \infty$. For any $c>1$ there is $C$ such that $\kappa_{n,\left[c k_{n}\right]}^{2} \geq C \kappa_{n}^{2}$ for all $n$.

Example. Let

$$
\kappa_{n j}^{2}=n^{-\lambda} \frac{1}{j^{\gamma}+c n^{\beta}}, \quad \gamma>1,
$$

with $\lambda=2-2 r-\beta$ and $\beta=(2-4 r) \gamma$. Then A1 - A5 hold.

Note that A1-A5 imply

$$
\kappa_{n}^{4}=\kappa_{n k_{n}}^{4} \asymp n^{-2} k_{n}^{-1} \quad \text { and } \quad k_{n} \asymp n^{2-4 r} .
$$

Theorems 4.1 - 4.10 given below represent realization of program announced in Introduction.

\subsection{Analytic form of necessary and sufficient conditions of consistency}

The results will be provided in terms of Fourier coefficients of functions $f_{n}=\sum_{j=1}^{\infty} \theta_{n j} \phi_{j}$.

Theorem 4.1. Assume A1-A5. Sequence of alternatives $f_{n}, \mathrm{cn}^{-r} \leq\left\|f_{n}\right\| \leq C n^{-r}$, is consistent, if and only if, there are $c_{1}, c_{2}$ and $n_{0}$ such that there holds

$$
\sum_{|j|<c_{2} k_{n}}\left|\theta_{n j}\right|^{2}>c_{1} n^{-2 r}
$$

for all $n>n_{0}$.

Versions of Theorems 4.1, 4.2 and 4.8 hold for setups of other sections. In setups of these sections indices $j$ may accept negative values and $\theta_{n j}$ may be complex numbers. By this reason we write $|j|$ instead of $j$ and $\left|\theta_{n j}\right|$ instead of $\theta_{n j}$ in (4.3), (4.4) and (4.9).

Theorem 4.2. Assume A1-A5. Sequence of alternatives $f_{n}, \mathrm{cn}^{-r} \leq\left\|f_{n}\right\| \leq \mathrm{Cn} n^{-r}$, is inconsistent, if and only if, for any $c_{2}$, there holds

$$
\sum_{|j|<c_{2} k_{n}}\left|\theta_{n j}\right|^{2}=o\left(n^{-2 r}\right) \quad \text { as } \quad n \rightarrow \infty .
$$

Proof of Theorems is based on Theorem 4.3 on asymptotic minimaxity of test statistics $T_{n}$.

Define sequence of tests $K_{n}\left(Y_{n}\right)=\mathbf{1}_{\left\{n^{-1} T_{n}\left(Y_{n}\right)>\left(2 A_{n}\right)^{1 / 2} x_{\alpha}\right\}}, 0<\alpha<1$, where $x_{\alpha}$ is defined by the equation $\alpha=1-\Phi\left(x_{\alpha}\right)$. 
Theorem 4.3. Assume A1-A5. Then sequence of tests $K_{n}\left(Y_{n}\right)$ is asymptotically minimax for the sets $\Upsilon_{n}\left(R_{n}, c\right)$ of alternatives. There hold $\alpha\left(K_{n}\right)=\alpha+o(1)$ and

$$
\beta\left(K_{n}, f_{n}\right)=\Phi\left(x_{\alpha}-R_{n}\left(f_{n}\right)\left(2 A_{n}\right)^{-1 / 2}\right)(1+o(1))
$$

uniformly onto all sequences $f_{n}$ such that $R_{n}\left(f_{n}\right)<C$ for any $C>0$.

A version of Theorem 4.3 for the problem of signal detection with heteroscedastic white noise has been proved in [8].

Such a form of conditions in Theorems 4.1 and 4.2 can be explained by concentration of coefficients $\kappa_{n j}^{2}$ in zone $j=O\left(k_{n}\right)$ for test statistics $T_{n}$ and for $A_{n}\left(\boldsymbol{\theta}_{n}\right)$.

Version of Theorem 4.3 for problem of hypothesis testing on distribution function provides necessary and sufficient conditions of uniform consistency of sets of alternatives defined in terms of distribution functions.

\subsection{Maxisets. Qualitative structure of consistent sequences of alternatives}

Denote $s=\frac{r}{2-4 r}$. Then $r=\frac{2 s}{1+4 s}$.

Theorem 4.4. Assume A1-A5. Then balls $\overline{\mathbb{B}}_{2 \infty}^{s}\left(P_{0}\right), P_{0}>0$, are maxisets for test statistics $T_{n}\left(Y_{n}\right)$.

For maxisets $\overline{\mathbb{B}}_{2 \infty}^{s}\left(P_{0}\right)$ with deleted "small" $\mathbb{L}_{2^{-}}$ball asymptotically minimax tests have been found in [11]. In [16], similar result has been obtained for Besov bodies in $\mathbb{B}_{2 \infty}^{s}$ defined in terms of wavelets coefficients.

Theorem 4.5. Assume A1-A5. Then sequence of alternatives $f_{n}, \mathrm{cn}^{-r} \leq\left\|f_{n}\right\| \leq$ $C n^{-r}$, is consistent, if and only if, there are maxiset $\overline{\mathbb{B}}_{2 \infty}^{s}\left(P_{0}\right), P_{0}>0$, and sequence $f_{1 n} \in \overline{\mathbb{B}}_{2 \infty}^{s}\left(P_{0}\right), c_{1} n^{-r} \leq\left\|f_{1 n}\right\| \leq C_{1} n^{-r}$, such that $f_{1 n}$ is orthogonal to $f_{n}-f_{1 n}$, that is, there holds

$$
\left\|f_{n}\right\|^{2}=\left\|f_{1 n}\right\|^{2}+\left\|f_{n}-f_{1 n}\right\|^{2}
$$

Therefore, if we have maxiset $\overline{\mathbb{B}}_{2 \infty}^{s}\left(P_{0}\right), P_{0}>0$, sequence of arbitrary functions $f_{1 n} \in$ $\overline{\mathbb{B}}_{2 \infty}^{s}\left(P_{0}\right), c_{1} n^{-r} \leq\left\|f_{1 n}\right\| \leq C_{1} n^{-r}$ and sequence of arbitrary functions $f_{2 n}, c_{1} n^{-r} \leq$ $\left\|f_{2 n}\right\| \leq C_{1} n^{-r}$ orthogonal to $f_{1 n}$, then sequence of simple alternatives $f_{n}=f_{1 n}+f_{2 n}$ is consistent.

Theorem 4.6. Assume A1-A5. Then, for any $\varepsilon>0$, for any consistent sequence of alternatives $f_{n}, c n^{-r} \leq\left\|f_{n}\right\| \leq C n^{-r}$ there are maxiset $\overline{\mathbb{B}}_{2 \infty}^{s}\left(P_{0}\right), P_{0}>0$, and sequence of functions $f_{1 n}, c_{1} n^{-r} \leq\left\|f_{1 n}\right\| \leq C_{1} n^{-r}$, belonging to maxiset $\bar{B}_{2 \infty}^{s}\left(P_{0}\right)$ such that there holds

function $f_{1 n}$ is orthogonal to $f_{n}-f_{1 n}$ 
for any $\alpha, 0<\alpha<1$, for the tests $K_{n}, \alpha\left(K_{n}\right)=\alpha(1+o(1))$ as $n \rightarrow \infty$, there is $n_{\varepsilon}$ such that, for any $n>n_{\varepsilon}$, there hold

$$
\left|\beta\left(K_{n}, f_{n}\right)-\beta\left(K_{n}, f_{1 n}\right)\right| \leq \varepsilon
$$

and

$$
\beta\left(K_{n}, f_{n}-f_{1 n}\right) \geq 1-\alpha-\varepsilon .
$$

If functions $f_{n}=\sum_{j=1}^{\infty} \theta_{n j} \phi_{j}$ satisfy $c_{1} n^{-r} \leq\left\|f_{n}\right\| \leq C_{1} n^{-r}$, then for any $c$ there is $P_{0}$ such that $f_{1 n}=\sum_{j=1}^{\left[c k_{n}\right]} \theta_{n j} \phi_{j} \in \overline{\mathbb{B}}_{2 \infty}^{s}\left(P_{0}\right)$ (see Lemma A.4). Since coefficients $\kappa_{n j}^{2}$, $j>c k_{n}$, are relatively small for large $c$, this allows to prove Theorems 4.5 and 4.6.

Maxisets $\overline{\mathbb{B}}_{2 \infty}^{s}\left(P_{0}\right), P_{0}>0$ in Theorems $4.5,4.6$ and 4.9 can be replaced with arbitrary maxiset $U$.

\subsection{Interaction of consistent and inconsistent sequences of alternatives. Purely consistent sequences}

Theorem 4.7. Assume A1-A5. Let sequence of alternatives $f_{n}$ be consistent. Then, for any inconsistent sequence of alternatives $f_{1 n}$, for tests $K_{n}, \alpha\left(K_{n}\right)=\alpha(1+o(1))$, $0<\alpha<1$, generated test statistics $T_{n}$, there holds

$$
\lim _{n \rightarrow \infty}\left(\beta\left(K_{n}, f_{n}\right)-\beta\left(K_{n}, f_{n}+f_{1 n}\right)\right)=0 .
$$

Theorem 4.8. Assume A1-A5. Sequence of alternatives $f_{n}, \mathrm{cn}^{-r} \leq\left\|f_{n}\right\| \leq C n^{-r}$, is purely $n^{-r}$-consistent, if and only if, for any $\varepsilon>0$, there is $C_{1}=C_{1}(\varepsilon)$ such that there holds

$$
\sum_{|j|>C_{1} k_{n}}\left|\theta_{n j}\right|^{2} \leq \varepsilon n^{-2 r}
$$

for all $n>n_{0}(\varepsilon)$.

Theorem 4.9. Assume A1-A5. Then sequence $f_{n}, \mathrm{cn}^{-r} \leq\left\|f_{n}\right\| \leq C n^{-r}$, is purely $n^{-r}$-consistent, if and only if, for any $\varepsilon>0$, there is $\gamma_{\epsilon}$ and sequence of functions $f_{1 n}$ belonging to maxiset $\overline{\mathbb{B}}_{2 \infty}^{s}\left(\gamma_{\epsilon}\right)$ such that $\left\|f_{n}-f_{1 n}\right\| \leq \varepsilon n^{-r}$ and (4.6) holds.

Theorem 4.10. Assume A1-A5. Then sequence of alternatives $f_{n}, c n^{-r}<\left\|f_{n}\right\|<$ $C n^{-r}$, is purely $n^{-r}$-consistent, if and only if, for any inconsistent subsequence of alternatives $f_{1 n_{i}}, c n_{i}^{-r}<\left\|f_{1 n_{i}}\right\|<C n_{i}^{-r}$, there holds

$$
\left\|f_{n_{i}}+f_{1 n_{i}}\right\|^{2}=\left\|f_{n_{i}}\right\|^{2}+\left\|f_{1 n_{i}}\right\|^{2}+o\left(n_{i}^{-r}\right),
$$

where $n_{i} \rightarrow \infty$ as $i \rightarrow \infty$. 
Remark 4.1. Let $\kappa_{n j}^{2}>0$ for $j \leq l_{n}$ and let $\kappa_{n j}^{2}=0$ for $j>l_{n}$ with $l_{n} \asymp n^{2-4 r}$ as $n \rightarrow \infty$. Analysis of proofs of Theorems shows that Theorems $4.1-4.10$ remain valid for this setup if A4 and A5 are replaced with

A6. For any $c, 0<c<1$, there is $c_{1}$ such that $\kappa_{n,\left[c l_{n}\right]}^{2} \geq c_{1} \kappa_{n 1}^{2}$ for all $n$.

In all corresponding reasoning we should put $\kappa_{n}^{2}=\kappa_{n 1}^{2}$ and $k_{n}=l_{n}$.

Theorems 4.2 and 4.8 hold with the following changes. It suffices to put $c_{2}<1$ in Theorem 4.2 and to take $C_{1}(\epsilon)<1$ in Theorem 4.8.

Proof of corresponding versions of Theorems $4.1-4.10$ is obtained by simplification of provided reasoning and is omitted.

\section{Kernel-based tests}

We continue to explore problem (1.6) and (1.7) of signal detection in Gaussian white noise with $0<r<1 / 2$. We suppose additionally that signal $f$ belongs to $\mathbb{L}_{2}^{\text {per }}\left(\mathbb{R}^{1}\right)$ the set of 1-periodic functions such that $f(t) \in \mathbb{L}_{2}(0,1)$. This allows to extend our model on real line $\mathbb{R}^{1}$ putting $w(t+j)=w(t)$ for all integer $j$ and $t \in[0,1)$ and to write the forthcoming integrals over all real line.

Define kernel estimator

$$
\hat{f}_{n}(t)=\frac{1}{h_{n}} \int_{-\infty}^{\infty} K\left(\frac{t-u}{h_{n}}\right) d Y_{n}(u), \quad t \in(0,1),
$$

where $h_{n}>0, h_{n} \rightarrow 0$ as $n \rightarrow \infty$.

The kernel $K$ is bounded function such that the support of $K$ is contained in $[-1,1]$, $K(t)=K(-t)$ for $t \in \mathbb{R}^{1}$ and $\int_{-\infty}^{\infty} K(t) d t=1$.

Denote $K_{h}(t)=\frac{1}{h} K\left(\frac{t}{h}\right), t \in \mathbb{R}^{1}$ and $h>0$.

In (5.1) we supposed that, for any $v, 0<v<1$, we have

$$
\int_{1}^{1+v} K_{h_{n}}(t-u) d Y_{n}(u)=\int_{0}^{v} K_{h_{n}}(t-1-u) f(u) d u+\frac{\sigma}{\sqrt{n}} \int_{0}^{v} K_{h_{n}}(t-1-u) d w(u)
$$

and

$$
\int_{-v}^{0} K_{h_{n}}(t-u) d Y_{n}(u)=\int_{1-v}^{1} K_{h_{n}}(t-u+1) f(u) d u+\frac{\sigma}{\sqrt{n}} \int_{1-v}^{1} K_{h_{n}}(t-u+1) d w(u) .
$$

Define kernel-based test statistics

$$
T_{n}\left(Y_{n}\right)=T_{n h_{n}}\left(Y_{n}\right)=n h_{n}^{1 / 2} \sigma^{-2} \gamma^{-1}\left(\left\|\hat{f}_{n}\right\|^{2}-\sigma^{2}\left(n h_{n}\right)^{-1}\|K\|^{2}\right),
$$

where

$$
\gamma^{2}=2 \int_{-\infty}^{\infty}\left(\int_{-\infty}^{\infty} K(t-s) K(s) d s\right)^{2} d t
$$


We call sequence of alternatives $f_{n}, c n^{-r} \leq\left\|f_{n}\right\| \leq C n^{-r}, n^{-r}$-consistent if, there is constant $c_{1}$ such that (2.1) holds for any tests $K_{n}, \alpha\left(K_{n}\right)=\alpha(1+o(1)) .0<\alpha<1$, generated sequence of test statistics $T_{n}$ with $h_{n}<c_{1} n^{4 r-2}, h_{n} \asymp n^{4 r-2}$.

We call sequence of alternatives $f_{n}, c n^{-r} \leq\left\|f_{n}\right\| \leq C n^{-r}, n^{-r}$-inconsistent if sequence of alternatives $f_{n}$ is inconsistent for any tests generated arbitrary test statistics $T_{n}$ with $h_{n} \rightarrow 0$ as $n \rightarrow \infty$.

Problem will be explored in terms of sequence model.

Let we observe a realization of random process $Y_{n}(t)$ with $f=f_{n}$.

For $-\infty<j<\infty$, denote

$$
\begin{gathered}
\hat{K}(j h)=\int_{-1}^{1} \exp \{2 \pi i j t\} K_{h}(t) d t, \quad h>0, \\
y_{n j}=\int_{0}^{1} \exp \{2 \pi i j t\} d Y_{n}(t), \quad \xi_{j}=\int_{0}^{1} \exp \{2 \pi i j t\} d w(t), \\
\theta_{n j}=\int_{0}^{1} \exp \{2 \pi i j t\} f_{n}(t) d t .
\end{gathered}
$$

In this notation we can write kernel estimator in the following form

$$
\hat{\theta}_{n j}=\hat{K}\left(j h_{n}\right) y_{n j}=\hat{K}\left(j h_{n}\right) \theta_{n j}+\sigma n^{-1 / 2} \hat{K}\left(j h_{n}\right) \xi_{j}, \quad-\infty<j<\infty,
$$

and test statistics $T_{n}$ admit the following representation

$$
T_{n}\left(Y_{n}\right)=n h_{n}^{1 / 2} \sigma^{-2} \gamma^{-1}\left(\sum_{j=-\infty}^{\infty}\left|\hat{\theta}_{n j}\right|^{2}-n^{-1} \sigma^{2} \sum_{j=-\infty}^{\infty}\left|\hat{K}\left(j h_{n}\right)\right|^{2}\right) .
$$

If we put $\left|\hat{K}\left(j h_{n}\right)\right|^{2}=\kappa_{n j}^{2}$, we get that definitions of test statistics $T_{n}\left(Y_{n}\right)$ in this section and in sections 4 are almost coincide. The setup of section 5 differs from setup of section 4 only heteroscedastic white noise. Another difference in the setup is that the function $\hat{K}(\omega), \omega \in \mathbb{R}^{1}$, may have zeros. Since differences are insignificant the same results are valid. Denote $k_{n}=\left[n^{2-4 r}\right]$.

Theorem 5.1. The statements of Theorems 4.1, 4.2, 4.5-4.10 hold for this setup as well. The statement of Theorem 4.4 holds also with $\overline{\mathbb{B}}_{2 \infty}^{s}$ replaced with $\mathbb{B}_{2 \infty}^{s}$.

In version of Theorem 4.4, ii. in definition of maxisets holds for test statistics $T_{n}$ having arbitrary values $h_{n}>0, h_{n} \rightarrow 0$ as $n \rightarrow \infty$.

Denote

$$
T_{1 n}(f)=T_{1 n}\left(f, h_{n}\right)=\int_{0}^{1}\left(\frac{1}{h_{n}} \int K\left(\frac{t-s}{h_{n}}\right) f(s) d s\right)^{2} d t .
$$

For sequence $\rho_{n}>0$, define sets

$$
\Upsilon_{n h_{n}}\left(T_{1 n}, \rho_{n}\right)=\left\{f: T_{1 n}(f)>\rho_{n}, f \in \mathbb{L}_{2}^{\text {per }}\left(\mathbb{R}^{1}\right)\right\} .
$$


Define sequence of kernel-based tests $K_{n}=\mathbf{1}_{\left\{T_{n}\left(Y_{n}\right) \geq x_{\alpha}\right\}}, 0<\alpha<1$, with $x_{\alpha}$ defined the equation $\alpha=1-\Phi\left(x_{\alpha}\right)$.

Proof of Theorems is based on the following Theorem 5.2 on asymptotic minimaxity of kernel-based tests $K_{n}$ (see Theorem 2.1.1 in [8]).

Theorem 5.2. Let $h_{n}^{-1 / 2} n^{-1} \rightarrow 0, h_{n} \rightarrow 0$ as $n \rightarrow \infty$. Let

$$
0<\liminf _{n \rightarrow \infty} n \rho_{n} h_{n}^{1 / 2} \leq \limsup _{n \rightarrow \infty} n \rho_{n} h_{n}^{1 / 2}<\infty .
$$

Then sequence of kernel-based tests $K_{n}$, is asymptotically minimax for the sets of alternatives $\Upsilon_{n h_{n}}\left(T_{1 n}, \rho_{n}\right)$. There hold $\alpha\left(L_{n}\right)=\alpha(1+o(1))$ and

$$
\beta\left(K_{n}, f_{n}\right)=\Phi\left(x_{\alpha}-\gamma^{-1} \sigma^{-2} n h_{n}^{1 / 2} T_{1 n}\left(f_{n}\right)\right)(1+o(1))
$$

uniformly onto sequences $f_{n} \in \mathbb{L}_{2}^{\text {per }}\left(R^{1}\right)$ such that $n h_{n}^{1 / 2} T_{1 n}\left(f_{n}\right)<C$.

We have

$$
T_{1 n}\left(f_{n}\right)=\sum_{j=-\infty}^{\infty}\left|\hat{K}\left(j h_{n}\right)\right|^{2}\left|\theta_{n j}\right|^{2} .
$$

Note that the unique difference of setups of Theorems 5.2 and 4.3 is heteroscedastic noise. Thus roof of Theorem 5.2 can be obtained by easy modification of the proof of Theorem 4.3 .

\section{6. $\chi^{2}$-tests}

Let $X_{1}, \ldots, X_{n}$ be i.i.d.r.v.'s having c.d.f. $F \in \Im$. Let c.d.f. $F(x)$ have a density $1+f(x)=$ $d F(x) / d x, x \in(0,1), f \in L_{2}^{p e r}(0,1)$.

We explore the problem of testing hypothesis (1.6) versus alternatives (1.7) with $0<$ $r<1 / 2$ discussed in Introduction.

For any sequence $m_{n}$, denote $\hat{p}_{n j}=\hat{F}_{n}\left(j / m_{n}\right)-\hat{F}_{n}\left((j-1) / m_{n}\right), 1 \leq j \leq m_{n}$.

Test statistics of $\chi^{2}$-tests equal

$$
T_{n}\left(\hat{F}_{n}\right)=n m_{n} \sum_{j=1}^{m_{n}}\left(\hat{p}_{n j}-1 / m_{n}\right)^{2} .
$$

Let

$$
f_{n}=\sum_{j=-\infty}^{\infty} \theta_{n j} \phi_{j}, \quad \phi_{j}(x)=\exp \{2 \pi i j x\}, \quad x \in(0,1) .
$$

We call sequence of alternatives $f_{n}, c n^{-r} \leq\left\|f_{n}\right\| \leq C n^{-r}, n^{-r}$-consistent, if there is $c_{1}$ such that, (2.1) holds for any tests $K_{n}, \alpha\left(K_{n}\right)=\alpha(1+o(1)) .0<\alpha<1$, generated sequence of chi-squared test statistics $T_{n}$ with number of cells $m_{n}>c_{1} n^{2-4 r}, m_{n} \asymp n^{2-4 r}$. 
We call sequence of alternatives $f_{n}, c n^{-r} \leq\left\|f_{n}\right\| \leq C n^{-r}, n^{-r}$-inconsistent if sequence of alternatives $f_{n}$ is inconsistent for all tests generated test statistics $T_{n}$ having number of cells $m_{n}, m_{n} \rightarrow \infty$ as $n \rightarrow \infty$..

Denote $k_{n}=\left[n^{\frac{2}{1+4 s}}\right] \asymp n^{2-4 r}$.

The differences in versions of Theorems $4.1-4.10$ for this setup are caused only the requirement that functions $f_{n}, f_{1 n}$ and $f_{2 n}$ should be densities.

Theorem 6.1. The statements of Theorems 4.1, 4.2 and 4.4-4.6, 4.8-4.10 hold for this setup with the following differences.

In version of Theorem 4.4 balls $\overline{\mathbb{B}}_{2 \infty}^{s}$ is replaced with bodies $\tilde{\mathbb{B}}_{2 \infty}^{s}$.

In version of Theorem 4.4, ii. in definition of maxisets holds for test statistics $T_{n}$ with arbitrary choice of number of cells $m_{n}, m_{n} \rightarrow \infty$ as $n \rightarrow \infty$.

In version of Theorem 4.6 we consider only sequences of alternatives $f_{n}$ such that the following assumption holds.

B. There is $c_{0}$ such that, for all $c>c_{0}$, functions

$$
1+f_{c n}=1+\sum_{|j|>c m_{n}} \theta_{j} \phi_{j} \quad \text { and } \quad 1+f_{n}-f_{c n}=1+\sum_{|j|<c m_{n}} \theta_{j} \phi_{j}
$$

are densities. B.

We implement definition of purely consistent sequences only for sequences $f_{n}$ satisfying

In proof of version of Theorem 4.6 for chi-squared tests, we show that there is $C_{\varepsilon}=$ $C\left(\varepsilon, c, C, c_{0}\right)$ such that, for densities $1+f_{1 n}=1+\sum_{|j|<C_{\varepsilon} m_{n}} \theta_{j} \phi_{j}$, (4.6), (4.7) and (4.9) hold. By Lemma A.4 given below, there is $\gamma_{\varepsilon}$ such that $f_{1 n} \in \gamma_{\varepsilon} U$.

In Theorem 6.2, given bellow, definitions of consistency and inconsistency proposed in subsection 2.1 are treated if simple alternatives $f_{n}$ are replaced with distribution functions $F_{n}$ and hypothesis is $\mathbb{H}_{0}: F(x)=F_{0}(x)=x, x \in[0,1]$.

Theorem 6.2. Let sequence of alternatives $F_{n}$ be consistent. Let $F_{1 n}$ be inconsistent sequence of alternatives such that $F_{2 n}=F_{n}(x)+F_{1 n}(x)-F_{0}(x)$ are distribution functions. Then for tests $K_{n}, \alpha\left(K_{n}\right)=\alpha(1+o(1)), 0<\alpha<1$, there holds

$$
\lim _{n \rightarrow \infty}\left(\beta\left(K_{n}, F_{n}\right)-\beta\left(K_{n}, F_{2 n}\right)\right)=0 .
$$

Proof of Theorems are based on the following Theorem 6.3 on asymptotic minimaxity of chi-squared tests given below. Theorem 6.3 is summary of results of Theorems 2.1 and 2.4 in [7].

For sequence $\rho_{n}>0$, define sets of alternatives

$$
\Upsilon_{n}\left(T_{n}, \rho_{n}\right)=\left\{F: T_{n}(F) \geq \rho_{n}, F \in \Im\right\}
$$

The definition of asymptotic minimaxity of tests is the same as in section 4 .

imsart-bj ver. 2014/10/16 file: nuconxi.tex date: September 1, 2020 
Define the tests

$$
K_{n}=\mathbf{1}_{\left\{2^{-1 / 2} m_{n}^{-1 / 2}\left(T_{n}\left(\hat{F}_{n}\right)-m_{n}+1\right)>x_{\alpha}\right\}}
$$

where $x_{\alpha}$ is defined the equation $\alpha=1-\Phi\left(x_{\alpha}\right)$.

Theorem 6.3. Let $m_{n} \rightarrow \infty, m_{n}^{-1} n^{2} \rightarrow \infty$ as $n \rightarrow \infty$. Let

$$
0<\liminf _{n \rightarrow \infty} m_{n}^{-1 / 2} \rho_{n} \leq \limsup _{n \rightarrow \infty} m_{n}^{-1 / 2} \rho_{n}<\infty .
$$

Then $\chi^{2}$-tests $K_{n}, \alpha\left(K_{n}\right)=\alpha+o(1), 0<\alpha<1$, are asymptotically minimax for the sets of alternatives $\Upsilon_{n}\left(T_{n}, \rho_{n}\right)$. There holds

$$
\beta\left(K_{n}, F_{n}\right)=\Phi\left(x_{\alpha}-2^{-1 / 2} m_{n}^{-1 / 2} T_{n}\left(F_{n}\right)\right)(1+o(1))
$$

uniformly onto sequences $F_{n}$ such that $T_{n}\left(F_{n}\right) \leq C m_{n}^{1 / 2}$.

Note that for implementation of Theorem 6.3 to proof of Theorems 6.1 and 6.2 we need to make a transition from indicator functions to trigonometric functions. Such a transition is realized in Appendix.

\section{Cramer - von Mises tests}

We consider Cramer - von Mises test statistics as functional

$$
T^{2}\left(\hat{F}_{n}-F_{0}\right)=\int_{0}^{1}\left(\hat{F}_{n}(x)-F_{0}(x)\right)^{2} d F_{0}(x)
$$

depending on empirical distribution function $\hat{F}_{n}$. Here $F_{0}(x)=x, x \in[0,1]$.

Denote $K_{n}=K_{n}\left(X_{1}, \ldots, X_{n}\right)$ sequence of Cramer- von Mises tests.

A part of further results holds for setup (1.1) and (1.2) with $\Upsilon_{n}=\Upsilon_{n}(a) \doteq \Upsilon_{n}\left(T^{2}, a n^{-1}\right)$, $a>0$.

We say that Cramer - von Mises test is asymptotically unbiased if, for any $a>0$, for any $\alpha, 0<\alpha<1$, for tests $K_{n}, \alpha\left(K_{n}\right)=\alpha+o(1)$, there holds

$$
\limsup _{n \rightarrow \infty} \sup _{F \in \Upsilon_{n}(a)} \beta_{F}\left(K_{n}\right)<1-\alpha .
$$

Nonparametric tests satisfying (7.1) are called also uniformly consistent (see Ch. 14.2 in [24]).

Proof of results is based on the following Theorem 7.1.

Theorem 7.1. The following three statements hold.

i. For sequence of alternatives $F_{n}$, there is sequence of Cramer - von Mises tests $K_{n}$ such that

$$
\lim _{n \rightarrow \infty}\left(\alpha\left(K_{n}\right)+\beta_{F_{n}}\left(K_{n}\right)\right)=0
$$


holds, if and only if, there holds

$$
\lim _{n \rightarrow \infty} n T^{2}\left(F_{n}-F_{0}\right)=\infty .
$$

ii. Cramer - von Mises tests are asymptotically unbiased.

iii. For any sequence of Cramer - von Mises tests $K_{n}$,

$$
\lim _{n \rightarrow \infty}\left(\alpha\left(K_{n}\right)+\beta_{F_{n}}\left(K_{n}\right)\right) \geq 1
$$

holds, iff, there holds

$$
\lim _{n \rightarrow \infty} n T^{2}\left(F_{n}-F_{0}\right)=0 .
$$

Sufficiency in i. and iii. in Theorem 7.1 is wellknown (see [15]). Necessary conditions in $i$. and in iii. follows easily from ii.

From now on we explore the problem of testing hypothesis (1.6) versus alternatives (1.7) with $0<r<1 / 2$ discussed in Introduction.

If c.d.f. $F$ has density, we can write the functional $T^{2}\left(F-F_{0}\right)$ in the following form (see Ch.5, [28])

$$
T^{2}\left(F-F_{0}\right)=\int_{0}^{1} \int_{0}^{1}(\min \{s, t\}-s t) f(t) f(s) d s d t
$$

with $f(t)=d\left(F(t)-F_{0}(t)\right) / d t$.

If we consider the orthonormal expansion of function

$$
f(t)=\sum_{j=1}^{\infty} \theta_{j} \phi_{j}(t)
$$

on trigonometric basis $\phi_{j}(t)=\sqrt{2} \cos (\pi j t), 1 \leq j<\infty$, then we get

$$
n T^{2}\left(F-F_{0}\right)=n \sum_{j=1}^{\infty} \frac{\theta_{j}^{2}}{\pi^{2} j^{2}}
$$

Denote $k_{n}=\left[n^{(1-2 r) / 2}\right]$.

In Theorems 7.2 and 7.3 given below, we follow the definition of consistency provided in subsection 2.1.

Theorem 7.2. For orthonormal system of functions $\phi_{j}(t)=\sqrt{2} \cos (\pi j t), t \in[0,1)$, $j=1,2, \ldots$, the bodies $\overline{\mathbb{B}}_{2 \infty}^{s}\left(P_{0}\right)$ with $s=\frac{2 r}{1-2 r}, r=\frac{s}{2+2 s}$, are maxisets for Cramer - von Mises test statistics.

In previous sections functionals $T_{n}$ depend on $n$. In this setup we explore the unique functional $T$ for all $n$ and for different values of $r, 0<r<1 / 2$. To separate the study 
of sequences of alternatives for different $r$, we consider for fixed $r$ only sequences of alternatives satisfying G1.

G1. For any $\varepsilon>0$ there is $c_{\epsilon}$ such that there holds

$$
n \sum_{|j|<c_{\epsilon} k_{n}} \theta_{n j}^{2} j^{-2}<\varepsilon
$$

for all $n>n_{0}\left(\varepsilon, c_{\epsilon}\right)$.

If G1 does not hold for some $c_{\epsilon}=c_{n} \rightarrow 0, c_{n} k_{n} \rightarrow \infty$ as $n \rightarrow \infty$ and functions $1+\bar{f}_{n}=1+\sum_{j<c_{n} k_{n}} \theta_{n j} \phi_{j}$ are densities, then (2.1) holds for some sequence of functions $\bar{f}_{n},\left\|\bar{f}_{n}\right\|=o\left(n^{-r}\right)$. Thus this case of consistency can be studied in the framework of the faster rate of convergence of sequence of alternatives.

Theorem 7.3. Let sequence of alternatives $f_{n}$ satisfies G1. Then for sequence $f_{n}$ the statements of Theorems 4.1, 4.2, 4.5, 4.6, 4.8 and 4.10 are valid with the following changes.

In version of Theorem 4.6 it is supposed that B holds.

In Theorem 7.3 definition of pure consistency is considered for sequences of functions $f_{n}$ satisfying $\mathrm{B}$.

Theorem 7.4. The statement of Theorem 6.2 holds for this setup as well.

\section{8. $n^{-1 / 2}-$ rate of convergence}

In section we extend results of sections $4-7$ to the case $r=1 / 2$. We show that, for $r=1 / 2$, the sets

$$
\left.U\left(l, P_{0}\right)\right)=\left\{f: f=\sum_{j=1}^{\infty} \theta_{j} \phi_{j},\|f\| \leq P_{0}, f \in \mathbb{L}_{2}(0,1)\right\}
$$

with $l=1,2, \ldots$ and $P_{0}>0$ and the linear space $\Xi=\left\{f: f \in U\left(l, P_{0}\right)\right.$ for some integer $l$ and $\left.P_{0}>0\right\}$ satisfy $i$. and $i$. respectively in definition of maxisets. Moreover sets $U\left(l, P_{0}\right)$ can replace with maxisets in versions of Theorems 4.5, 4.6 and 4.9 .

Problems of hypothesis testing in sections 4,5 and 7 are covered the following setup.

We observe sequence of independent random variables $y_{j}=\theta_{j}+n^{-1 / 2} \sigma_{j} \xi_{j}$ where $\xi_{j}$, $1 \leq j<\infty$, are Gaussian random variables, $\mathbf{E} \xi_{j}=0$ and $\mathbf{E}\left[\xi_{j}^{2}\right]=1$.

Define functional

$$
T(\boldsymbol{\theta})=\sum_{j=1}^{\infty} \kappa_{j}^{2} \theta_{j}^{2}, \quad \boldsymbol{\theta}=\left\{\theta_{j}\right\}_{1}^{\infty}
$$

where coefficients $\kappa_{j}^{2}$ satisfy the following conditions.

D1. Sequence $\kappa_{j}^{2}$ is decreasing and $\sum_{j=1}^{\infty} \kappa_{j}^{2}<\infty$. 
D2 There is $C>0$ such that $0<\sigma_{j}<C$ for all $1 \leq j<\infty$.

Problem is to test hypothesis

$$
\mathbb{H}_{0}: \theta_{j}=0, \quad 1 \leq j<\infty
$$

versus alternatives

$$
\mathbb{H}_{n}: \theta_{j}=\theta_{n j}, \quad 1 \leq j<\infty,
$$

where $T\left(\boldsymbol{\theta}_{n}\right) \asymp n^{-1}$ with $\boldsymbol{\theta}_{n}=\left\{\theta_{n j}\right\}_{1}^{\infty}$.

Theorem 8.1. For $r=1 / 2$ sets $U\left(l, P_{0}\right), l=1,2, \ldots, P_{0}>0$, and linear space $\Xi$ satisfy $i$. and ii. respectively in definition of maxisets.

Theorem 8.2. Assume D1 and D2. Then Theorems 4.1, 4.2 and 4.4 - 4.10 are valid with $k_{n}=1$ and sets $\overline{\mathbb{B}}_{2 \infty}^{s}\left(P_{0}\right)$ replaced with sets $U\left(l, P_{0}\right)$, where $l=1,2, \ldots$ and $P_{0}>0$.

Proof of Theorem 8.2 is based on Theorem 8.3 given below and evident modification of iii. in Theorem 7.1 on this setup. Reasoning are akin to proof of Theorems in section 4 and is omitted. Note only that for verifying ii in definition of maxisets we put $\bar{f}_{l}=\sum_{j=l}^{\infty} \theta_{j} \phi_{j}$ (see proof of Theorem 4.4). After that we implement version of Theorem 4.2 for this setup.

Denote $z_{j}=n^{1 / 2} y_{j}$ and $\eta_{j}=n^{1 / 2} \theta_{j}$. Then problem of hypothesis testing (8.1) and (8.2) is replaced with the following.

We observe independent random variables $z_{j}=\eta_{j}+\sigma_{j} \xi_{j}$. Problem is to test hypothesis

$$
\mathbb{H}_{0}: \eta_{j}=0, \quad 1 \leq j<\infty
$$

versus alternatives

$$
\mathbb{H}_{n}: \eta_{j}=\tau_{j}, \quad 1 \leq j<\infty,
$$

where $0<T(\boldsymbol{\tau})<\infty$ with $\boldsymbol{\tau}=\left\{\tau_{j}\right\}_{1}^{\infty}$.

For $a>0$, define sets of alternatives

$$
\Upsilon(a)=\left\{\boldsymbol{\eta}: T(\boldsymbol{\eta})>a, \boldsymbol{\eta}=\left\{\eta_{j}\right\}_{1}^{\infty}, \eta_{j} \in \mathbb{R}^{1}\right\}
$$

We say that test $K$ is unbiased [24], if

$$
\alpha(K)+\beta(K, \Upsilon(a))<1 .
$$

Denote $\boldsymbol{z}=\left\{z_{j}\right\}_{1}^{\infty}$.

Theorem 8.3. Assume D1 and D2. Then tests $K, \alpha(K)=\alpha, 0<\alpha<1$, generated test statistics $T(\boldsymbol{z})$ are unbiased.

Proof of Theorem 8.3 is provided in A.5.

For chi-squared tests with number of cells $m_{n}=m=$ const similar Theorem holds for $r=1 / 2$ with the same definition of consistency as in section 6 .

imsart-bj ver. 2014/10/16 file: nuconxi.tex date: September 1, 2020 
Theorem 8.4. For $r=1 / 2$, for chi-squared tests Theorem 8.1 holds as well. Statement of Theorems 4.1, 4.2 and 4.5, 4.6, 4.8-4.10 hold with the same changes as in Theorem 6.1 and with $k_{n}=1$.

Emphasize that Besov bodies $\tilde{\mathbb{B}}_{2 \infty}^{s}\left(P_{0}\right)$ in versions of Theorems $4.5,4.6$ and 4.9 are replaced with sets $U\left(l, P_{0}\right), l=1,2, \ldots$ and $P_{0}>0$.

For proof of Theorem 8.4 we implement wellknown fact that $n T_{n}\left(F_{n}\right)>c$ is necessary and sufficient condition for consistency of sequence of alternatives $F_{n} \in \Im$ for chi-squared tests with fixed number of cells.

Theorem 8.3 allows to obtain versions of Theorems $7.2-7.4$ for problem of hypothesis testing 8.3 and 8.4 with test statistics $T$ having $\kappa_{j}^{2} \asymp j^{-2 \lambda}, 2 \lambda>1$.

Such a setup arises in particular for test statistics $T$ constructed on the base of technique of reproducing kernel Hilbert spaces [13].

Theorem 8.5. Let $\kappa_{j}^{2} \asymp j^{-2 \lambda}, 2 \lambda>1$. Then statements of Theorems 7.2 - 7.4 holds with $s=\frac{2 \lambda r}{1-2 \lambda r}$ and $k_{n} \asymp n^{\frac{1-2 r}{2 \lambda}}$ as $n \rightarrow \infty$. All assumptions caused the requirement of density non-negativity are omitted.

Proof of Theorem 8.5 is akin to proof of Theorems $7.2-7.4$ and is omitted.

\section{Appendix A: Proof of Theorems}

\section{A.1. Proof of Theorems of section 3}

It suffices to prove only necessary conditions.

We suppose set $U$ is closed. General setup can be reduced easily to this one.

First we prove Theorem 3.1 if set $U$ is center-symmetric.

We remind that set $U$ is center-symmetric if $\boldsymbol{\theta} \in U$ implies $-\boldsymbol{\theta} \in U$.

Lemma A.1. Suppose that set $U$ is bounded, convex and center-symmetric. Then the statement of Theorem 3.1 holds.

For any vectors $\boldsymbol{\theta}_{1} \in \mathbb{H}$ and $\boldsymbol{\theta}_{2} \in \mathbb{H}$ define segment $\operatorname{int}\left(\boldsymbol{\theta}_{1}, \boldsymbol{\theta}_{2}\right)=\left\{\boldsymbol{\theta}: \boldsymbol{\theta}=(1-\lambda) \boldsymbol{\theta}_{1}+\right.$ $\left.\lambda \boldsymbol{\theta}_{2}, \lambda \in[0,1]\right\}$.

Proof of Lemma A.1 is based on the following Lemma A.2.

Lemma A.2. For any vectors $\boldsymbol{\theta}_{1} \in U$ and $\boldsymbol{\theta}_{2} \in U$ we have int $\left(\frac{\boldsymbol{\theta}_{1}-\boldsymbol{\theta}_{2}}{2}, \frac{\boldsymbol{\theta}_{2}-\boldsymbol{\theta}_{1}}{2}\right) \subset U$. There holds $0 \in \mathfrak{i n t}\left(\frac{\boldsymbol{\theta}_{1}-\boldsymbol{\theta}_{2}}{2}, \frac{\boldsymbol{\theta}_{2}-\boldsymbol{\theta}_{1}}{2}\right)$ and segment $\mathfrak{i n t}\left(\frac{\boldsymbol{\theta}_{1}-\boldsymbol{\theta}_{2}}{2}, \frac{\boldsymbol{\theta}_{2}-\boldsymbol{\theta}_{1}}{2}\right)$ is parallel to segment $\mathfrak{i n t}\left(\boldsymbol{\theta}_{1}, \boldsymbol{\theta}_{2}\right)$.

Remark 3.1. Let we have segment $\mathfrak{i n t}\left(\boldsymbol{\theta}_{1}, \boldsymbol{\theta}_{2}\right) \subset U$. Let $\boldsymbol{\eta}$ and $\boldsymbol{-} \boldsymbol{\eta}$ be the points of intersection of the line $L=\left\{\boldsymbol{\theta}: \boldsymbol{\theta}=\lambda\left(\boldsymbol{\theta}_{1}-\boldsymbol{\theta}_{2}\right), \lambda \in \mathbb{R}^{1}\right\}$ and the boundary of set $U$. Then, by Lemma A.2, we get $\left\|\boldsymbol{\theta}_{1}-\boldsymbol{\theta}_{2}\right\| \leq 2\|\boldsymbol{\eta}\|$. 
Proof of Lemma A.2. . Segments int $\left(\boldsymbol{\theta}_{1}, \boldsymbol{\theta}_{2}\right) \subset U$ and $\mathfrak{i n t}\left(-\boldsymbol{\theta}_{1},-\boldsymbol{\theta}_{2}\right) \subset U$ are parallel. For each $\lambda \in[0,1]$ we have $(1-\lambda) \boldsymbol{\theta}_{1}+\lambda \boldsymbol{\theta}_{2} \in \mathfrak{i n t}\left(\boldsymbol{\theta}_{1}, \boldsymbol{\theta}_{2}\right)$ and $-\lambda \boldsymbol{\theta}_{1}-(1-$ $\lambda) \boldsymbol{\theta}_{2} \in \mathfrak{i n t}\left(-\boldsymbol{\theta}_{1},-\boldsymbol{\theta}_{2}\right)$. The middle $\boldsymbol{\theta}_{\lambda}=\left((1-2 \lambda) \boldsymbol{\theta}_{1}-(1-2 \lambda) \boldsymbol{\theta}_{2}\right) / 2$ of segment $\mathfrak{i n t}\left((1-\lambda) \boldsymbol{\theta}_{1}+\lambda \boldsymbol{\theta}_{2},-\lambda \boldsymbol{\theta}_{1}-(1-\lambda) \boldsymbol{\theta}_{2}\right) \subset U$ belongs to segment int $\left(\frac{\boldsymbol{\theta}_{1}-\boldsymbol{\theta}_{2}}{2}, \frac{\boldsymbol{\theta}_{2}-\boldsymbol{\theta}_{1}}{2}\right)$ and, for each point $\boldsymbol{\theta}$ of segment $\operatorname{int}\left(\frac{\boldsymbol{\theta}_{1}-\boldsymbol{\theta}_{2}}{2}, \frac{\boldsymbol{\theta}_{2}-\boldsymbol{\theta}_{1}}{2}\right)$, there is $\lambda \in[0,1]$ such that $\boldsymbol{\theta}=\boldsymbol{\theta}_{\lambda}$. Therefore $\operatorname{int}\left(\frac{\boldsymbol{\theta}_{1}-\boldsymbol{\theta}_{2}}{2}, \frac{\boldsymbol{\theta}_{2}-\boldsymbol{\theta}_{1}}{2}\right) \subset U$.

Proof of Lemma A.1. Define sequence of orthogonal vectors $\boldsymbol{e}_{i}$ by induction.

Define vector $\boldsymbol{e}_{1}, \boldsymbol{e}_{1} \in U$, such that $\left\|\boldsymbol{e}_{1}\right\|=\sup \{\|\boldsymbol{\theta}\|, \boldsymbol{\theta} \in U\}$. Denote $\Pi_{1}$ linear subspace generated $\boldsymbol{e}_{1}$. Denote $\Gamma_{1}$ linear subspace orthogonal to $\Pi_{1}$.

Define vector $\boldsymbol{e}_{i} \in U \cap \Gamma_{i-1}$ such that $\left\|\boldsymbol{e}_{i}\right\|=\sup \left\{\|\boldsymbol{\theta}\|: \boldsymbol{\theta} \in U \cap \Gamma_{i-1}\right\}$. Denote $\Pi_{i}$ linear subspace generated vectors $\boldsymbol{e}_{1}, \ldots, \boldsymbol{e}_{i}$. Denote $\Gamma_{i}$ linear subspace orthogonal to $\Pi_{i}$.

Denote $d_{i}=\left\|\boldsymbol{e}_{i}\right\|$. Note that $d_{i} \rightarrow 0$ as $i \rightarrow \infty$. Otherwise, by Theorem 5.3 in [10], there does not exist uniformly consistent tests for the problem of testing hypothesis $\mathbb{H}_{0}: \boldsymbol{\theta}=\mathbf{0}$ versus alternative $\mathbb{H}_{1}: \boldsymbol{\theta}=\boldsymbol{e}_{i}, i=1,2, \ldots$

For any $\varepsilon \in(0,1)$ denote $l_{\varepsilon}=\min \left\{j: d_{j}<\varepsilon, j=1,2, \ldots\right\}$.

Denote $B_{r}(\boldsymbol{\theta})$ ball having radius $r$ and center $\boldsymbol{\theta}$.

It suffices to show that, for any $\varepsilon_{1}>0$, there is finite coverage of set $U$ by balls $B_{\varepsilon_{1}}(\boldsymbol{\theta})$.

Denote $\varepsilon=\varepsilon_{1} / 9$.

Denote $U_{\varepsilon}$ projection of set $U$ onto subspace $\Pi_{l_{\varepsilon}}$.

Denote $\tilde{B}_{r}(\boldsymbol{\theta})$ ball in $\Pi_{l_{\varepsilon}}$ having radius $r$ and center $\boldsymbol{\theta} \in \Pi_{l_{\varepsilon}}$. There is ball $\tilde{B}_{\delta_{1}}(0)$ such that $\tilde{B}_{\delta_{1}}(0) \subset U$. Denote $\delta=\min \left\{\varepsilon, \delta_{1}\right\}$.

Let $\boldsymbol{\theta}_{1}, \ldots, \boldsymbol{\theta}_{k}$ be $\delta$-net in $U_{\varepsilon}$.

Let $\boldsymbol{\eta}_{1}, \ldots, \boldsymbol{\eta}_{k}$ be points of $U$ such that $\boldsymbol{\theta}_{i}$ is projection of $\boldsymbol{\eta}_{i}$ onto subspace $\Pi_{l_{\varepsilon}}$ for $1 \leq i \leq k$.

Let us show that $B_{\varepsilon_{1}}\left(\boldsymbol{\eta}_{1}\right), \ldots, B_{\varepsilon_{1}}\left(\boldsymbol{\eta}_{k}\right)$ is coverage of set $U$.

Let $\boldsymbol{\eta} \in U$ and let $\boldsymbol{\theta}$ be projection of $\boldsymbol{\eta}$ onto $\Pi_{l_{\varepsilon}}$. There is $i, 1 \leq i \leq k$, such that $\left\|\boldsymbol{\theta}_{i}-\boldsymbol{\theta}\right\| \leq \delta$. It suffices to show that $\boldsymbol{\eta} \in B_{\varepsilon_{1}}\left(\boldsymbol{\eta}_{i}\right)$.

By Lemma A.2, int $\left(\frac{\boldsymbol{\eta}_{i}-\boldsymbol{\eta}}{2}, \frac{\boldsymbol{\eta}-\boldsymbol{\eta}_{i}}{2}\right) \subset U$. Since $\boldsymbol{\theta}_{i}-\boldsymbol{\theta} \in \Pi_{l_{\epsilon}}$ and $\boldsymbol{\theta}_{i}-\boldsymbol{\theta} \in \tilde{B}_{\delta}(\mathbf{0})$, then $\left(\boldsymbol{\theta}_{i}-\boldsymbol{\theta}\right) / 2 \in U$. Since set $U$ is center-symmetric and convex we have $\frac{1}{2}\left(\left(\boldsymbol{\eta}_{i}-\boldsymbol{\eta}\right) / 2\right)-$ $\frac{1}{2}\left(\left(\boldsymbol{\theta}_{i}-\boldsymbol{\theta}\right) / 2\right) \in U$. Note that vector $\left(\boldsymbol{\eta}_{i}-\boldsymbol{\theta}_{i}\right)-(\boldsymbol{\eta}-\boldsymbol{\theta})$ is orthogonal to the subspace $\Pi_{l_{\varepsilon}}$. Therefore $\left\|\left(\left(\boldsymbol{\eta}_{i}-\boldsymbol{\theta}_{i}\right)-(\boldsymbol{\eta}-\boldsymbol{\theta})\right) / 4\right\| \leq 2 \varepsilon$. Therefore $\left\|\boldsymbol{\eta}-\boldsymbol{\eta}_{i}\right\| \leq 8 \varepsilon+\left\|\boldsymbol{\theta}-\boldsymbol{\theta}_{i}\right\|<9 \varepsilon$. This implies $\boldsymbol{\eta} \in B_{\varepsilon_{1}}\left(\boldsymbol{\eta}_{i}\right)$.

Proof of Theorem 3.1. We say that set $\bar{W}$ is trimmed symmetrization of set $W$ if $\boldsymbol{x} \in \bar{W}$ holds, if and only if, $\boldsymbol{x} \in W$ and $-\boldsymbol{x} \in W$. If $W$ is convex, then $\bar{W}$ is convex as well.

Since $\bar{U} \subset U$, then there is consistent tests for problem of testing hypothesis $\boldsymbol{\theta}=\mathbf{0}$ versus alternatives $\overline{\mathbb{H}}_{n}: \boldsymbol{\theta} \in \bar{V}_{n}=\left\{\boldsymbol{\theta}:\|\boldsymbol{\theta}\| \geq \rho_{n}, \boldsymbol{\theta} \in \bar{U}\right\}$ if there is consistent test for sets of alternatives $V_{n}$.

Therefore set $\bar{U}$ is compact. We show that this implies that set $U$ is compact as well. 
Suppose otherwise. Then there are points $\boldsymbol{x}_{i} \in U, 1 \leq i<\infty$, and positive constant $b$ such that $\rho\left(\boldsymbol{x}_{i}, \Pi_{i-1}\right)>b$ for $2 \leq i<\infty$. Here $\Pi_{i-1}$ is hyperplane generated points $\boldsymbol{x}_{1}, \ldots, \boldsymbol{x}_{i-1}$. Then convex hull $L \subset U$ of points $\boldsymbol{x}_{1}, \boldsymbol{x}_{2}, \ldots$ is not compact as well.

Denote $M$ hyperplane generated by points $\boldsymbol{x}_{i}, 1 \leq i<\infty$. Without loss of generality we can suppose $\mathbf{0} \notin M$. There is $\lambda<0$ such that $\boldsymbol{x}_{0}=\lambda \boldsymbol{w} \in U$ where $\boldsymbol{w}=\frac{\boldsymbol{x}_{1}+\boldsymbol{x}_{2}}{2}$.

Denote $K$ convex hull of points $\boldsymbol{x}_{0}, \boldsymbol{x}_{1}, \boldsymbol{x}_{2}, \ldots$. Let $\bar{K}$ be trimmed symmetrization of $K$. Then $\bar{K} \subset \bar{U}$ and therefore $\bar{K}$ is compact. Let us show that there is $\delta>0$ such that $\boldsymbol{x}_{0}+\delta\left(\boldsymbol{x}_{i}-\boldsymbol{x}_{0}\right) \in \bar{K}$ for all $i=1,2, \ldots$. Therefore set of points $\boldsymbol{x}_{0}+\delta\left(\boldsymbol{x}_{i}-\boldsymbol{x}_{0}\right)$, $i=1,2, \ldots$, is compact. We will come to contradiction.

Denote $d=\sup \{\|\boldsymbol{x}-\boldsymbol{y}\|: \boldsymbol{x}, \boldsymbol{y} \in U\}$.

Denote $\alpha_{k}$ angle between vectors $\boldsymbol{x}_{k}-\boldsymbol{x}_{0}$ and $\boldsymbol{w}-\boldsymbol{x}_{0}$.

Denote $\beta_{k}$ angle between vectors $\boldsymbol{w}-\boldsymbol{x}_{k}$ and $\boldsymbol{w}-\boldsymbol{x}_{0}$.

Then angle $\gamma_{k}$ between vectors $\boldsymbol{x}_{0}-\boldsymbol{x}_{0}$ and $\boldsymbol{w}-\boldsymbol{x}_{0}$ equals $\beta_{k}-\alpha_{k}$.

If we show $\gamma_{k}>c>0$ for all $k$, we prove the existence $\delta>0$.

Denote $\boldsymbol{w}_{k}$ projection of $\boldsymbol{x}_{k}$ on a line passing through points $\boldsymbol{x}_{0}$ and $\boldsymbol{w}$.

Then $\left\|\boldsymbol{x}_{k}-\boldsymbol{w}\right\| \geq b$ and $\left\|\boldsymbol{x}_{0}-\boldsymbol{w}_{k}\right\| \leq\left\|\boldsymbol{x}_{0}-\boldsymbol{x}_{k}\right\| \leq d$.

Hence we have

$$
\begin{aligned}
& \gamma_{k}=\arctan \frac{\left\|\boldsymbol{x}_{k}-\boldsymbol{w}_{k}\right\|}{\left\|\boldsymbol{w}-\boldsymbol{w}_{k}\right\|}-\arctan \frac{\left\|\boldsymbol{x}_{k}-\boldsymbol{w}_{k}\right\|}{\left\|\boldsymbol{x}_{0}-\boldsymbol{w}_{k}\right\|} \\
& \geq \arctan \frac{b}{d-\left\|\boldsymbol{x}_{0}-\boldsymbol{w}\right\|}-\arctan \frac{b}{d}>c>0 .
\end{aligned}
$$

Proof of Theorem 3.3. . Proof of Theorem 3.1 is based on Theorem 5.3 in [10]. For linear inverse ill-posed problems (3.4), Theorem 5.5 in [10] is akin to Theorem 5.3 in [10]. Thus it suffices to implement Theorem 5.5 in [10] instead of Theorem 5.3 in [10] in proof of Theorem 3.1.

\section{A.2. Proof of Theorems of section 4}

Proof of Theorem 4.3. Theorem 4.3 and its version for Remark 4.1 setup can be deduced straightforwardly from Theorem 1 in [6].

Lower bound follows from reasoning of Theorem 1 in [6] straightforwardly.

Upper bound follows from the following reasoning. We have

$$
\begin{aligned}
& \sum_{j=1}^{\infty} \kappa_{n j}^{2} y_{j}^{2}=\sum_{j=1}^{\infty} \kappa_{n j}^{2} \theta_{n j}^{2}+2 \frac{\sigma}{\sqrt{n}} \sum_{j=1}^{\infty} \kappa_{n j}^{2} \theta_{n j} \xi_{j}+\frac{\sigma^{2}}{n} \sum_{j=1}^{\infty} \kappa_{n j}^{2} \xi_{j}^{2} \\
& =n^{-2} A_{n}\left(\boldsymbol{\theta}_{n}\right)+2 J_{1 n}+J_{2 n}
\end{aligned}
$$

with

$$
\mathbf{E}\left[J_{2 n}\right]=\frac{\sigma^{2}}{n} \rho_{n}, \quad \operatorname{Var}\left[J_{2 n}\right]=2 \frac{\sigma^{4}}{n^{4}} A_{n}
$$


and

$$
\operatorname{Var}\left[J_{1 n}\right]=\frac{\sigma^{2}}{n} \sum_{j=1}^{\infty} \kappa_{n j}^{4} \theta_{n j}^{2} \leq \frac{\sigma^{2} \kappa_{n}^{2}}{n} \sum_{j=1}^{\infty} \kappa_{n j}^{2} \theta_{n j}^{2}=o\left(n^{-4} A_{n}\left(\boldsymbol{\theta}_{n}\right)\right) .
$$

By Chebyshov inequality, it follows from (A.1) - (A.3), that, if $A_{n}=o\left(A_{n}\left(\boldsymbol{\theta}_{n}\right)\right)$ as $n \rightarrow \infty$, then $\beta\left(L_{n}, f_{n}\right) \rightarrow 0$ as $n \rightarrow \infty$. Thus it suffices to explore the case

$$
A_{n} \asymp A_{n}\left(\boldsymbol{\theta}_{n}\right)=n^{2} \sum_{j=1}^{\infty} \kappa_{n j}^{2} \theta_{n j}^{2} .
$$

If (A.4) holds, then, implementing the reasoning of proof of Lemma 1 in [6], we get that (4.5) holds.

Proof of Theorem 4.1. Let (4.3) hold. Then, by A5 and (4.2), we have

$$
A_{n}\left(\boldsymbol{\theta}_{n}\right)=n^{2} \sum_{j=1}^{\infty} \kappa_{n j}^{2} \theta_{n j}^{2} \geq C n^{2} \kappa_{n}^{2} \sum_{j=1}^{c_{2} k_{n}} \theta_{n j}^{2} \asymp n^{2} \kappa_{n}^{2} n^{-2 r} \asymp 1 .
$$

By Theorem 4.3, this implies sufficiency.

Necessary conditions follows from sufficiency conditions in Theorem 4.2.

Proof of Theorem 4.2. Let (4.4) hold. Then, by (4.2) and A2, we have

$$
A_{n}\left(\boldsymbol{\theta}_{n}\right) \leq C n^{2} \kappa_{n}^{2} \sum_{j<c_{2} k_{n}} \theta_{n j}^{2}+C n^{2} \kappa_{n,\left[c_{2} n\right]}^{2} \sum_{j>c_{2} n} \theta_{n j}^{2} \asymp o(1)+O\left(\kappa_{n,\left[c_{2} n\right]}^{2} / \kappa_{n}^{2}\right) .
$$

By A4, we have

$$
\lim _{c_{2} \rightarrow \infty} \lim _{n \rightarrow \infty} \kappa_{n,\left[c_{2} n\right]}^{2} / \kappa_{n}^{2} \rightarrow 0
$$

By Theorem 4.3, (A.5) and (A.6) together, we get sufficiency.

Proof of Theorem 4.4. Statement $i$. follows from Theorem 4.1 and Lemma A.3 provided below.

Lemma A.3. Let $f_{n} \in \overline{\mathbb{B}}_{2 \infty}^{s}\left(c_{1}\right)$ and $c n^{-r} \leq\left\|f_{n}\right\| \leq C n^{-r}$. Then, for $l_{n}=C_{1} n^{2-4 r}(1+$ $o(1))=C_{1} n^{\frac{r}{s}}(1+o(1))$ with $C_{1}^{2 s}>2 c_{1} / c$, there holds

$$
\sum_{j=1}^{l_{n}} \theta_{n j}^{2}>\frac{c}{2} n^{-2 r}(1+o(1))
$$

Proof. Let $f_{n} \in c_{1} U$. Then we have

$$
l_{n}^{2 s} \sum_{j=l_{n}}^{\infty} \theta_{n j}^{2}=C_{1}^{2 s} n^{2 r} \sum_{j=l_{n}}^{\infty} \theta_{n j}^{2}(1+o(1)) \leq c_{1}(1+o(1)) .
$$


Hence

$$
\sum_{j=l_{n}}^{\infty} \theta_{n j}^{2} \leq c_{1} C_{1}^{-2 s} n^{-2 r} \leq \frac{c}{2} n^{-2 r}(1+o(1))
$$

Therefore (A.7) holds.

Proof of Theorem 4.4 . Suppose opposite that ii. does not valid. Then $f=\sum_{j=1}^{\infty} \tau_{j} \phi_{j} \notin$ $\overline{\mathbb{B}}_{2 \infty}^{s}$. This implies that there is sequence $m_{l}, m_{l} \rightarrow \infty$ as $l \rightarrow \infty$, such that

$$
m_{l}^{2 s} \sum_{j=m_{l}}^{\infty} \tau_{j}^{2}=C_{l}
$$

with $C_{l} \rightarrow \infty$ as $l \rightarrow \infty$.

Define a sequence $\boldsymbol{\eta}_{l}=\left\{\eta_{l j}\right\}_{j=1}^{\infty}$ such that $\eta_{l j}=0$ if $j<m_{l}$ and $\eta_{l j}=\tau_{j}$ if $j \geq m_{l}$.

Since $V$ is convex and ortho-symmetric we have $\tilde{f}_{l}=\sum_{j=1}^{\infty} \eta_{l j} \phi_{j} \in V$.

For alternatives $\tilde{f}_{l}$ we define sequence $n_{l}$ such that

$$
\left\|\boldsymbol{\eta}_{l}\right\|^{2} \asymp n_{l}^{-2 r} \asymp m_{l}^{-2 s} C_{l} .
$$

Then

$$
n_{l} \asymp C_{l}^{-1 /(2 r)} m_{l}^{s / r}=C_{l}^{-1 /(2 r)} m_{l}^{\frac{1}{2-4 r}} .
$$

Therefore we get

$$
m_{l} \asymp C_{l}^{(1-2 r) / r} n_{l}^{2-4 r} .
$$

By A4, (A.12) implies

$$
\kappa_{n_{l} m_{l}}^{2}=o\left(\kappa_{n_{l}}^{2}\right)
$$

Using (4.2), A2 and (A.13), we get

$$
\begin{aligned}
& A_{n_{l}}\left(\boldsymbol{\eta}_{l}\right)=n_{l}^{2} \sum_{j=1}^{\infty} \kappa_{n_{l} j}^{2} \eta_{j l}^{2} \leq n_{l}^{2} \kappa_{m_{l} n_{l}}^{2} \sum_{j=m_{l}}^{\infty} \theta_{n_{l} j}^{2} \\
& \asymp n_{l}^{2-2 r} \kappa_{n_{l} m_{l}}^{2}=O\left(\kappa_{n_{l} m_{l}}^{2} \kappa_{n_{l}}^{-2}\right)=o(1) .
\end{aligned}
$$

By Theorem 4.3, (A.14) implies $n_{l}^{-r}$-inconsistency of sequence of alternatives $\tilde{f}_{l}$.

Proof of Theorem 4.5. Theorem 4.5 follows from Lemmas A.4 - A.6.

Lemma A.4. For any $c$ and any $C$ there is $\overline{\mathbb{B}}_{2 \infty}^{s}\left(P_{0}\right)$ such that, if $f_{n}=\sum_{j=1}^{c k_{n}} \theta_{n j} \phi_{j}$, and $\left\|f_{n}\right\| \leq C n^{-r}$, then $f_{n} \in \overline{\mathbb{B}}_{2 \infty}^{s}\left(P_{0}\right)$.

Proof. Let $C_{1}$ be such that $k_{n}=C_{1} n^{r / s}(1+o(1))$. Then we have

$$
k_{n}^{2 s} \sum_{j=1}^{c k_{n}} \theta_{n j}^{2} \leq C_{1} n^{2 r} \sum_{j=1}^{\infty} \theta_{n j}^{2}(1+o(1))<C C_{1}(1+o(1)) .
$$


Lemma A.5. Necessary conditions in Theorem 4.5 are fulfilled.

Proof. Let $f_{n}=\sum_{j=1}^{\infty} \theta_{n j} \phi_{j}$ and let $f_{1 n}=\sum_{j=1}^{\infty} \eta_{n j} \phi_{j}$. Denote $\zeta_{n j}=\theta_{n j}-\eta_{n j}, 1 \leq$ $j<\infty$.

For any $\delta>0, c_{1}$ and $C_{2}$, there is $c_{2}$ such that, for each sequence $f_{1 n} \in \overline{\mathbb{B}}_{2 \infty}^{s}\left(P_{0}\right)$, $\left\|f_{1 n}\right\| \leq C_{2} n^{-r}$, there holds

$$
\sum_{j>c_{2} k_{n}} \eta_{n j}^{2}<\delta n^{-2 r} .
$$

To prove (A.15) it suffices to put $c_{2} k_{n}=l_{n}=C_{1} n^{2-4 r}(1+o(1))$ in (A.8) with $C_{1}^{2 s}>\delta c_{1}$.

We have

$$
\begin{aligned}
& J_{n}=\left|\sum_{j>c k_{n}} \theta_{n j}^{2}-\sum_{j>c k_{n}} \zeta_{n j}^{2}\right| \leq \sum_{j>c k_{n}}\left|\eta_{n j}\left(2 \theta_{n j}-\eta_{j n}\right)\right| \\
& \leq\left(\sum_{j>c k_{n}} \eta_{n j}^{2}\right)^{1 / 2}\left(2\left(\sum_{j>c k_{n}} \theta_{n j}^{2}\right)^{1 / 2}+\left(\sum_{j>c k_{n}} \eta_{n j}^{2}\right)^{1 / 2}\right) \leq C \delta^{1 / 2} n^{-2 r} .
\end{aligned}
$$

By (4.6), using (A.15) and (A.16), we get

$$
\begin{aligned}
& \sum_{j<c k_{n}} \theta_{n j}^{2}=\sum_{j=1}^{\infty} \eta_{n j}^{2}+\sum_{j=1}^{\infty} \zeta_{n j}^{2}-\sum_{j \geq c k_{n}} \theta_{n j}^{2} \geq \sum_{j<c k_{n}} \eta_{n j}^{2}-J_{n} \\
& \geq \sum_{j<c k_{n}} \eta_{n j}^{2}-C \delta^{1 / 2} n^{-2 r} \geq\left\|f_{1 n}\right\|^{2}-\delta n^{-2 r}-C \delta^{1 / 2} n^{-2 r} .
\end{aligned}
$$

By Theorem 4.1, (A.17) implies consistency of sequence $f_{n}$.

Lemma A.6. Let sequence of alternatives $f_{n}, \mathrm{cn}^{-r} \leq\left\|f_{n}\right\| \leq C n^{-r}$, be consistent. Then (4.6) holds.

Proof. By Theorem 4.1, there are $c_{1}$ and $c_{2}$ such that sequence $f_{1 n}=\sum_{j<c_{2} k_{n}} \theta_{n j} \phi_{j}$ is consistent and $\left\|f_{1 n}\right\| \geq c_{1} n^{-r}$. By Lemma A.4, there is $\overline{\mathbb{B}}_{2 \infty}^{s}\left(P_{0}\right)$ such that $f_{1 n} \in$ $\overline{\mathbb{B}}_{2 \infty}^{s}\left(P_{0}\right)$.

Proof of Theorem 4.6. By A4 and (4.2), for any $\delta>0$, there is $c$ such that we have

$$
n^{2} \sum_{j>c k_{n}} \kappa_{n j}^{2} \theta_{n j}^{2} \leq \delta .
$$

By Lemma A.4, there is $P_{0}$ such that $f_{1 n}=\sum_{j<c k_{n}} \theta_{n j} \phi_{j} \in \overline{\mathbb{B}}_{2 \infty}^{s}\left(P_{0}\right)$. By Theorem 4.3 and (A.18), for sequence of alternatives $f_{1 n},(4.7)$ and (4.8) hold. 
Proof of Theorem 4.7. Let $f_{n}=\sum_{j=1}^{\infty} \theta_{n j} \phi_{j}$ and let $f_{1 n}=\sum_{j=1}^{\infty} \eta_{n j} \phi_{j}$. Denote $\boldsymbol{\eta}_{n}=\left\{\eta_{n j}\right\}_{j=1}^{\infty}$.

By Cauchy inequality, we have

$$
\begin{aligned}
& \left|A_{n}\left(\boldsymbol{\theta}_{n}\right)-A_{n}\left(\boldsymbol{\theta}_{n}+\boldsymbol{\eta}_{n}\right)\right|=n^{2}\left|\sum_{j=1}^{\infty} \kappa_{n j}^{2} \theta_{n j}^{2}-\sum_{j=1}^{\infty} \kappa_{n j}^{2}\left(\theta_{n j}+\eta_{n j}\right)^{2}\right| \\
& \leq 2 A_{n}^{1 / 2}\left(\boldsymbol{\theta}_{n}\right) A_{n}^{1 / 2}\left(\boldsymbol{\eta}_{n}\right)+A_{n}\left(\boldsymbol{\eta}_{n}\right) .
\end{aligned}
$$

By Theorem 4.3, inconsistency of sequence $f_{1 n}$ implies $A_{n}\left(\boldsymbol{\eta}_{n}\right)=o(1)$ as $n \rightarrow \infty$. Therefore, by (A.19), $\left|A_{n}\left(\boldsymbol{\theta}_{n}\right)-A_{n}\left(\boldsymbol{\theta}_{n}+\boldsymbol{\eta}_{n}\right)\right|=o(1)$ as $n \rightarrow \infty$. Hence, by Theorem 4.3, we get Theorem 4.7.

Proof of Theorem 4.8 . For proof of sufficiency suppose opposite. Then there is sequence $n_{i}, n_{i} \rightarrow \infty$ as $i \rightarrow \infty$ such that $f_{n_{i}}=f_{1 n_{i}}+f_{2 n_{i}}$,

$$
\left\|f_{n_{i}}\right\|^{2}=\left\|f_{1 n_{i}}\right\|^{2}+\left\|f_{2 n_{i}}\right\|^{2}
$$

$c_{1} n_{i}^{-r}<\left\|f_{1 n_{i}}\right\|<C_{1} n_{i}^{-r}, c_{2} n_{i}^{-r}<\left\|f_{2 n_{i}}\right\|<C_{2} n_{i}^{-r}$ and sequence $f_{2 n_{i}}$ is inconsistent.

Let $f_{n_{i}}=\sum_{j=1}^{\infty} \theta_{n_{i} j} \phi_{j}, f_{1 n_{i}}=\sum_{j=1}^{\infty} \theta_{1 n_{i} j} \phi_{j}$ and $f_{2 n_{i}}=\sum_{j=1}^{\infty} \theta_{2 n_{i} j} \phi_{j}$.

Then, by Theorem 4.2 and by (4.9), we get that there are $\varepsilon_{i}, \varepsilon_{i} \rightarrow 0$ and $C_{i}=C\left(\varepsilon_{i}\right)$, $C_{i} \rightarrow \infty$ as $i \rightarrow \infty$ such that

$$
\sum_{j>C_{i} k_{n}} \theta_{n_{i} j}^{2}=\sum_{j>C_{i} k_{n}}\left(\theta_{1 n_{i} j}+\theta_{2 n_{i} j}\right)^{2}=o\left(n^{-2 r}\right), \quad \sum_{j<C_{i} k_{n}} \theta_{2 n_{i} j}^{2}=o\left(n^{-2 r}\right) .
$$

By (A.20) and (A.21), we get

$$
\sum_{j=1}^{\infty} \theta_{n_{i} j}^{2}=\sum_{j<C_{i} k_{n}} \theta_{n_{i} j}^{2}+o\left(n^{-2 r}\right)=\sum_{j<C_{i} k_{n}} \theta_{1 n_{i} j}^{2}+o\left(n^{-2 r}\right) .
$$

Hence, by (A.20), we get $\left\|f_{2 n_{i}}\right\|=o\left(n^{-r}\right)$. We come to contradiction.

To prove necessary conditions suppose (4.9) does not hold. Then there are $\varepsilon>0$ and sequences $C_{i} \rightarrow \infty, n_{i} \rightarrow \infty$ as $i \rightarrow \infty$ such that

$$
\sum_{j>C_{i} k_{n_{i}}} \theta_{n_{i} j}^{2}>\varepsilon n_{i}^{-2 r}
$$

Then, by A4 and (4.2), we get

$$
n_{i}^{2} \sum_{j>C_{i} k_{n_{i}}} \kappa_{n_{i} j}^{2} \theta_{n_{i} j}^{2}=o(1)
$$

Therefore, by Theorem 4.3, subsequence $f_{1 n_{i}}=\sum_{j>C_{i} k_{n_{i}}} \theta_{n_{i} j} \phi_{j}$ is inconsistent. 
Proof of Theorem 4.9. For proof of necessary conditions, it suffices to put

$$
f_{1 n}=\sum_{j<C_{1}(\epsilon) k_{n}} \theta_{n j} \phi_{j}
$$

By Lemma A.4, there is $P_{0}>0$ such that $f_{1 n} \in \overline{\mathbb{B}}_{2 \infty}^{s}\left(P_{0}\right)$. Proof of sufficiency is simple and is omitted.

Proof of Theorem 4.10. Necessary conditions are rather evident, and proof is omitted. Proof of sufficiency is also simple.

Lemma A.7. Let for sequence $f_{n}, \mathrm{cn}^{-r}<\left\|f_{n}\right\|<\mathrm{Cn}^{-r}$, (4.10) hold. Then sequence $f_{n}$ is purely $n^{-r}$-consistent.

Suppose $f_{n}=\sum_{j=1}^{\infty} \theta_{n j} \phi_{j}$ is not purely $n^{-r}$-consistent. Then, by Theorem 4.8 , there are $c_{1}$ and sequences $n_{i}$, and $c_{n_{i}}, c_{n_{i}} \rightarrow \infty$ as $i \rightarrow \infty$, such that

$$
\sum_{j>c_{n_{i}} k_{n_{i}}} \theta_{n_{l} j}^{2}>c_{1} n_{i}^{-r}
$$

Therefore, if we put $f_{1 n_{i}}=\sum_{j>c_{n_{i}} k_{n_{i}}} \theta_{n_{i} j} \phi_{j}$, then (4.10) does not hold.

\section{A.3. Proof of Theorems of section 5}

Proof of version of Theorem 4.1. . Since $\hat{K}(\omega)$ is analytical function and $\hat{K}(0)=$ 1 there is $b>0$ such that $|\hat{K}(\omega)|>c>0$ for $|\omega|<b$.

Let (4.3) hold. Then we have

$$
\begin{aligned}
& T_{1 n}\left(f_{n}\right)=\sum_{j=-\infty}^{\infty}\left|\hat{K}\left(j h_{n}\right)\right|^{2}\left|\theta_{n j}\right|^{2} \geq \sum_{|j| h_{n}<b}\left|\hat{K}\left(j h_{n}\right)\right|^{2}\left|\theta_{n j}\right|^{2} \\
& \asymp \sum_{|j|<c_{2} k_{n}}\left|\hat{K}\left(j h_{n}\right)\right|^{2}\left|\theta_{n j}\right|^{2} \asymp n^{-1} h_{n}^{-1 / 2} \asymp n^{-2 r}
\end{aligned}
$$

for $c_{2} k_{n}<b h_{n}^{-1}$. By Theorem 5.2, this implies consistency.

Proof of version of Theorem 4.4. We verify only iv.. Let $f=\sum_{j=-\infty}^{\infty} \tau_{j} \phi_{j} \notin \mathbb{B}_{2 \infty}^{s}$. Then there is sequence $m_{l}, m_{l} \rightarrow \infty$ as $l \rightarrow \infty$, such that

$$
m_{l}^{2 s} \sum_{|j| \geq m_{l}}^{\infty}\left|\tau_{j}\right|^{2}=C_{l}
$$

with $C_{l} \rightarrow \infty$ as $l \rightarrow \infty$. 
It is clear that we can define a sequence $m_{l}$ such that

$$
m_{l}^{2 s} \sum_{m_{l} \leq|j| \leq 2 m_{l}}\left|\tau_{j}\right|^{2}>\delta C_{l}
$$

where $\delta, 0<\delta<1 / 2$, does not depend on $l$.

Otherwise, we have

$$
2^{2 s(i-1)} m_{l}^{2 s} \sum_{j=2^{i-1} m_{l}}^{2^{i} m_{l}} \tau_{j}^{2}<\delta C_{l}
$$

for all $i=1,2, \ldots$, that implies that the left hand-side of (A.23) does not exceed $2 \delta C_{l}$.

Define a sequence $\boldsymbol{\eta}_{l}=\left\{\eta_{l j}\right\}_{j=-\infty}^{\infty}$ such that $\eta_{l j}=\tau_{j}$ if $|j| \geq m_{l}$, and $\eta_{l j}=0$ otherwise.

Denote

$$
\tilde{f}_{l}(x)=\sum_{j=-\infty}^{\infty} \eta_{l j} \exp \{2 \pi i j x\}
$$

For alternatives $\tilde{f}_{l}(x)$ we define sequence $n_{l}$ such that $\left\|\tilde{f}_{l}(x)\right\| \asymp n_{l}^{-r}$.

Then

$$
n_{l} \asymp C_{l}^{-1 /(2 r)} m_{l}^{s / r} \text {. }
$$

We have $|\hat{K}(\omega)| \leq \hat{K}(0)=1$ for all $\omega \in R^{1}$ and $|\hat{K}(\omega)|>c>0$ for all $|\omega|<b$. Hence, if we put $h_{l}=h_{n_{l}}=2^{-1} b^{-1} m_{l}^{-1}$, then, by (A.24), there is $C>0$ such that, for all $h>0$, there holds

$$
T_{1 n_{l}}\left(\tilde{f}_{l}, h_{l}\right)=\sum_{j=-\infty}^{\infty}\left|\hat{K}\left(j h_{l}\right) \eta_{l j}\right|^{2}>C \sum_{j=-\infty}^{\infty}\left|\hat{K}(j h) \eta_{l j}\right|^{2}=C T_{1 n_{l}}\left(\tilde{f}_{l}, h\right) .
$$

Thus we can choose $h=h_{l}$ for further reasoning.

By (A.24), we get

$$
T_{1 n_{l}}\left(\tilde{f}_{l}\right)=\sum_{|j|>m_{l}}\left|\hat{K}\left(j h_{l}\right) \eta_{l j}\right|^{2} \asymp \sum_{j=m_{l}}^{2 m_{l}}\left|\eta_{l j}\right|^{2} \asymp n_{l}^{-2 r} .
$$

If we put in estimates $(\mathrm{A} .11),(\mathrm{A} .12), k_{l}=\left[h_{n_{l}}^{-1}\right]$ and $m_{l}=k_{l}$, then we get

$$
h_{n_{l}}^{1 / 2} \asymp C_{l}^{(2 r-1) / 2} n_{l}^{2 r-1} .
$$

By (A.25) and (A.26), we get

$$
n_{l} T_{1 n_{l}}\left(\tilde{f}_{l}\right) h_{n_{l}}^{1 / 2} \asymp C_{l}^{-(1-2 r) / 2} .
$$

By Theorem 5.2, this implies inconsistency of sequence of alternatives $\tilde{f}_{l}$. 


\section{A.4. Proof of Theorems of section 6}

We have

$$
n^{-1} m_{n}^{-1} T_{n}(F)=\sum_{l=0}^{m_{n}-1}\left(\int_{l / m_{n}}^{(l+1) / m_{n}} f(x) d x\right)^{2} .
$$

Using representation $f(x)$ as Fourier series

$$
f(x)=\sum_{j=-\infty}^{\infty} \theta_{j} \exp \{2 \pi i j x\}
$$

we get

$$
\int_{l / m_{n}}^{(l+1) / m_{n}} f(x) d x=\sum_{j=-\infty}^{\infty} \frac{\theta_{j}}{2 \pi i j} \exp \left\{2 \pi i j l / m_{n}\right\}\left(\exp \left\{2 \pi i j / m_{n}\right\}-1\right)
$$

for $1 \leq l<m_{n}$.

In what follows, we shall use the following agreement $0 / 0=0$.

Lemma A.8. There holds

$$
n^{-1} m_{n}^{-1} T_{n}(F)=m_{n} \sum_{k=-\infty}^{\infty} \sum_{j \neq k m_{n}} \frac{\theta_{j} \bar{\theta}_{j-k m_{n}}}{4 \pi^{2} j\left(j-k m_{n}\right)}\left(2-2 \cos \left(2 \pi j / m_{n}\right)\right) .
$$

Proof of Lemma A.8. We have

$$
\begin{aligned}
& n^{-1} m_{n}^{-1} T_{n}(F)=\sum_{l=0}^{m_{n}-1}\left(\sum_{j \neq 0} \frac{\theta_{j}}{2 \pi i j} \exp \left\{2 \pi i j l / m_{n}\right\}\left(\exp \left\{2 \pi i j / m_{n}\right\}-1\right)\right) \\
& \times\left(\sum_{j \neq 0} \frac{-\bar{\theta}_{j}}{2 \pi i j} \exp \left\{-2 \pi i j l / m_{n}\right\}\left(\exp \left\{-2 \pi i j / m_{n}\right\}-1\right)\right)=J_{1}+J_{2}
\end{aligned}
$$

with

$$
\begin{aligned}
& J_{1}=\sum_{l=0}^{m_{n}-1} \sum_{k=-\infty}^{\infty} \sum_{j_{1}=j-k m_{n}} \frac{\theta_{j} \bar{\theta}_{j_{1}}}{4 \pi^{2} j j_{1}} \exp \{2 \pi i l k\} \\
& \times\left(\exp \left\{2 \pi i j / m_{n}\right\}-1\right)\left(\exp \left\{-2 \pi i j_{1} / m_{n}\right\}-1\right) \\
& =m_{n} \sum_{k=-\infty}^{\infty} \sum_{j=-\infty}^{\infty} \frac{\theta_{j} \bar{\theta}_{j-k m_{n}}}{4 \pi^{2} j\left(j-k m_{n}\right)}\left(2-2 \cos \left(2 \pi j / m_{n}\right)\right)
\end{aligned}
$$

and

$$
\begin{aligned}
& J_{2}=\sum_{l=0}^{m_{n}-1} \sum_{j \neq 0} \sum_{j_{1} \neq j-k m_{n}} \frac{\theta_{j} \bar{\theta}_{j_{1}}}{4 \pi^{2} j j_{1}} \exp \left\{2 \pi i\left(j-j_{1}\right) l / m_{n}\right\} \\
& \times\left(\exp \left\{2 \pi i j / m_{n}\right\}-1\right)\left(\exp \left\{-2 \pi i j_{1} / m_{n}\right\}-1\right)=0
\end{aligned}
$$


where $j_{1} \neq j-k m_{n}$ signifies that summation is performed over all $j_{1}$ such that $j_{1} \neq$ $j-k m_{n}$ for all integer $k$.

In the last equality of (A.30), we make use of the identity

$$
\sum_{l=0}^{m_{n}-1} \exp \left\{2 \pi i\left(j-j_{1}\right) l / m_{n}\right\}=\frac{\exp \left\{2 \pi i\left(j-j_{1}\right) m_{n} / m_{n}\right\}-1}{\exp \left\{2 \pi i\left(j-j_{1}\right) / m_{n}\right\}-1}=0,
$$

if $j-j_{1} \neq k m_{n}$ for all integer $k$.

By (A.28) - (A.30) together, we get (A.27).

For any c.d.f $F$ and any $k$ denote $\tilde{F}_{k}$ the function having the derivative

$$
1+\tilde{f}_{k}(x)=1+\sum_{|j|>k} \theta_{j} \exp \{2 \pi i j x\}
$$

and such that $\tilde{F}_{k}(1)=1$.

Denote $i_{n}=\left[d m_{n}\right]$ where $d>1+c$.

Lemma A.9. There holds

$$
n^{-1} m_{n}^{-2} T_{n}\left(\tilde{F}_{i_{n}}\right) \leq C m_{n}^{-1} i_{n}^{-1} \sum_{|j|>i_{n}}\left|\theta_{j}\right|^{2} .
$$

Proof. Denote $\eta_{j}=\theta_{j}$ if $|j|>i_{n}$ and $\eta_{j}=0$ if $|j|<i_{n}$.

We have

$$
\begin{aligned}
& n^{-1} m_{n}^{-2} T_{n}\left(\tilde{F}_{i_{n}}\right)=\sum_{k=-\infty}^{\infty} \sum_{j \neq k m_{n}} \frac{\eta_{j} \bar{\eta}_{j-k m_{n}}}{4 \pi^{2} j\left(j-k m_{n}\right)}\left(2-2 \cos \left(2 \pi j / m_{n}\right)\right) \\
& \leq C \sum_{|j|>i_{n}}\left|\frac{\eta_{j}}{j}\right| \sum_{k=-\infty}^{\infty}\left|\frac{\eta_{j+k m_{n}}}{j+k m_{n}}\right| \\
& =C \sum_{j=1}^{m_{n}} \sum_{k=-\infty}^{\infty}\left|\frac{\eta_{j+k m_{n}}}{j+k m_{n}}\right| \sum_{k_{1}=-\infty}^{\infty}\left|\frac{\eta_{j+\left(k+k_{1}\right) m_{n}}}{j+\left(k+k_{1}\right) m_{n}}\right| \\
& =C \sum_{j=1}^{m_{n}}\left(\sum_{k=-\infty}^{\infty}\left|\frac{\eta_{j+k m_{n}}}{j+k m_{n}}\right|\right)^{2} \\
& \leq C \sum_{j=1}^{m_{n}}\left(\sum_{|k|>d-1}\left|\eta_{j+k m_{n}}\right|^{2}\right)\left(\sum_{|k|>d-1}\left(j+k m_{n}\right)^{-2}\right) \\
& \leq C \sum_{j=-\infty}^{\infty}\left|\eta_{j}\right|^{2} \sum_{|k|>d}\left(k m_{n}\right)^{-2} \leq C m_{n}^{-1} i_{n}^{-1} \sum_{|j|>i_{n}}\left|\theta_{j}\right|^{2} .
\end{aligned}
$$


Proof of version of Theorem 4.1.. We prove sufficiency. Suppose (4.3) holds. Denote

$$
\tilde{f}_{n}=\tilde{f}_{n, c_{2} k_{n}}=\sum_{|j|>c_{2} k_{n}} \theta_{n j} \phi_{j} \quad \text { and } \quad \bar{f}_{n}=\bar{f}_{n, c_{2} k_{n}}=f_{n}-\tilde{f}_{n}
$$

Denote $\tilde{F}_{n}, \bar{F}_{n}$ the functions having derivatives $1+\tilde{f}_{n, c_{2} k_{n}}$ and $1+\bar{f}_{n, c_{2} k_{n}}$ respectively and such that $\tilde{F}_{n}(1)=1$ and $\bar{F}_{n}(1)=1$.

Let $T_{n}$ be chi-squared test statistics with a number of cells $m_{n}=\left[c_{3} k_{n}\right]$ where $c_{2}<c_{3}$. Denote $\mathbb{L}_{2, n}$ linear space generated functions $\mathbf{1}_{\left\{x \in\left((j-1) / m_{n}, j / m_{n}\right)\right\}}, 1 \leq j \leq m_{n}$.

Denote $\bar{h}_{n}$ orthogonal projection of $\bar{f}_{n}$ onto $\mathbb{L}_{2, n}$. Denote $\tilde{h}_{n}$ orthogonal projection of $\tilde{f}_{n}$ onto the line $\left\{h: h=\lambda \bar{h}_{n}, \lambda \in \mathbb{R}^{1}\right\}$.

Note that $n^{-1 / 2} T_{n}^{1 / 2}\left(F_{n}\right)$ equals the $\mathbb{L}_{2, n}$-norm of function $f_{n}$. Hence we have

$$
n^{-1 / 2} m_{n}^{-1} T_{n}^{1 / 2}\left(F_{n}\right) \geq\left\|\bar{h}_{n}+\tilde{h}_{n}\right\| .
$$

Thus, by Theorem 6.3 , it suffices to show that, for some choice of $c_{3}$, there holds $\| \bar{h}_{n}+$ $\tilde{h}_{n} \| \asymp n^{-r}$ if $m_{n}>c_{3} k_{n}$.

Denote $\bar{g}_{n}=\bar{f}_{n}-\bar{h}_{n}$ and $\tilde{g}_{n}=\tilde{f}_{n}-\tilde{h}_{n}$.

Denote

$$
\bar{p}_{j n}=\frac{1}{m_{n}} \int_{(j-1) / m_{n}}^{j / m_{n}} \bar{f}_{n}(x) d x, \quad 1 \leq j \leq m_{n} .
$$

By Lemmas 3 and 4 in section 7 of [31], we have

$$
\left\|\bar{g}_{n}\right\|^{2}=m_{n} \sum_{j=1}^{m_{n}} \int_{(j-1) / m_{n}}^{j / m_{n}}\left(\bar{f}_{n}(x)-\bar{p}_{j n}\right)^{2} d x \leq 2 \omega^{2}\left(\frac{1}{m_{n}}, \bar{f}_{n}\right) .
$$

Here

$$
\omega^{2}(h, f)=\int_{0}^{1}(f(t+h)-f(t))^{2} d t, \quad h>0,
$$

for any $f \in \mathbb{L}_{2}^{p e r}$. If $f=\sum_{j=-\infty}^{\infty} \theta_{j} \phi_{j}$, then

$$
\omega^{2}(s, f)=2 \sum_{j=1}^{\infty}\left|\theta_{j}\right|^{2}(2-2 \cos (2 \pi j s)) .
$$

Since $1-\cos (x) \leq x^{2}$, then, by (A.33) and (A.34), we have

$$
\left\|\bar{g}_{n}\right\| \leq 4 \pi\left(c_{2} k_{n} / m_{n}\right)^{1 / 2}\left\|\bar{f}_{n}\right\|=\delta\left\|\bar{f}_{n}\right\|(1+o(1)),
$$

where $\delta=4 \pi\left(c_{2} / c_{3}\right)^{1 / 2}$.

By (4.3), (A.33) and (A.35), we get that there is $c_{30}$, such that

$$
\left\|\bar{h}_{n}\right\|>\frac{c_{1}}{2} n^{-r}
$$


for $c_{3}>c_{30}$.

For any functions $g_{1}, g_{2} \in \mathbb{L}_{2}(0,1)$ denote $\left(g_{1}, g_{2}\right)$ inner product of $g_{1}$ and $g_{2}$.

We have

$$
0=\left(\bar{f}_{n}, \tilde{f}_{n}\right)=\left(\bar{h}_{n}, \tilde{h}_{n}\right)+\left(\bar{g}_{n}, \tilde{f}_{n}\right) .
$$

By (A.35), we get

$$
\left|\left(\bar{g}_{n}, \tilde{f}_{n}\right)\right| \leq\left\|\bar{g}_{n}\right\|\left\|\tilde{f}_{n}\right\| \leq \delta C^{2} n^{-2 r}
$$

Therefore we get

$$
\left|\left(\bar{h}_{n}, \tilde{h}_{n}\right)\right| \leq \delta C^{2} n^{-2 r} .
$$

By (A.36) (A.38), we get that, for sufficiently small $\delta>0$, there holds $\left\|\bar{h}_{n}+\tilde{h}_{n}\right\| \asymp n^{-r}$. Hence, using (A.32) and implementing Theorem 6.3, we get sufficiency.

Proof of version of Theorem 4.2. We prove sufficiency. Let $k_{n}=\left[c_{1} n^{2-4 r}\right]$. For $c_{2}>2 c_{1}$, we have

$$
T_{n}^{1 / 2}\left(F_{n}\right) \leq T_{n}^{1 / 2}\left(\bar{F}_{n}\right)+T_{n}^{1 / 2}\left(\tilde{F}_{n}\right) .
$$

By Lemma A.9, we have

$$
n^{-1} m_{n}-2 T_{n}\left(\tilde{F}_{n}\right) \leq c_{2}^{-1} m_{n} k_{n}^{-1}\left\|\tilde{f}_{n}\right\|^{2} \leq c_{2}^{-1} c_{1} C n^{-2 r} .
$$

We have

$$
\left\|\bar{f}_{n}\right\| \geq n^{-1 / 2} m_{n}^{-1} T_{n}^{1 / 2}\left(\bar{F}_{n}\right) .
$$

Since one can take arbitrary value $c_{2}, c_{2}>2 c_{1}$, then, by Theorem 6.3, (4.4) and (A.39) - (A.41) together, we get inconsistency of sequence $f_{n}$.

Proof of version of Theorem 4.4. Let us prove ii. Suppose opposite. Then there is sequence $i_{l}, i_{l} \rightarrow \infty$ as $l \rightarrow \infty$, such that

$$
i_{l}^{2 s}\left\|\tilde{f}_{i_{l}}\right\|^{2}=C_{l},
$$

with $C_{l} \rightarrow \infty$ as $l \rightarrow \infty$. Here $f=\sum_{j=-\infty}^{\infty} \tau_{j} \phi_{j}$ and $\tilde{f}_{i_{l}}=\sum_{|j|>i_{l}} \tau_{j} \phi_{j}$.

Define sequence $n_{l}$ such that $n_{l}^{-r} \asymp\left\|\tilde{f}_{i_{l}}\right\|$ as $l \rightarrow \infty$.

Then, estimating similarly to (A.11) and (A.12), we get $i_{l}^{-1 / 2} \asymp C_{l}^{(2 r-1) / 2} n_{l}^{2 r-1}$ as $l \rightarrow \infty$.

If $m_{l}=o\left(i_{l}\right)$, then, by Lemma A.9, we get

$$
m_{l}^{-1 / 2} T_{n_{l}}\left(\tilde{F}_{i_{l}}\right) \leq m_{l}^{1 / 2} i_{l}^{-1} n_{l} \sum_{|j|>i_{l}}\left|\tau_{j}\right|^{2} \asymp m_{l}^{1 / 2} i_{l}^{-1} n_{l}^{1-2 r}=o\left(C_{l}^{(2 r-1) / 2}\right) .
$$

Let $m_{l} \asymp i_{l}$ or $i_{l}=o\left(m_{l}\right)$. Then we have

$$
n_{l}^{-2 r} \asymp\left\|\tilde{f}_{i_{l}}\right\|^{2} \geq n_{l}^{-1} m_{l}^{-2} T_{n_{l}}\left(\tilde{F}_{i_{l}}\right) .
$$

Therefore

$$
m_{l}^{-1 / 2} T_{n_{l}}\left(\tilde{F}_{i_{l}}\right) \leq C m_{l}^{-1 / 2} n_{l}^{1-2 r}=C m_{l}^{-1 / 2} i_{l}^{1 / 2} C_{l}^{(2 r-1) / 2}=o(1) .
$$

By Theorem 6.3, (A.42) -(A.44) imply ii. 
Proof of version of Theorem 4.6. Let $f_{1 n}=\sum_{|j|<c k_{n}} \theta_{n j} \phi_{j}$. Then, by Lemma A.4, there is maxiset $\tilde{\mathbb{B}}_{2 \infty}^{s}\left(P_{0}\right)$ such that $f_{1 n} \in \tilde{\mathbb{B}}_{2 \infty}^{s}\left(P_{0}\right)$.

Denote $F_{1 n}$ function having derivative $1+f_{1 n}$ and such that $F_{1 n}(1)=1$.

We have

$$
\left|T_{n}^{1 / 2}\left(F_{n}\right)-T_{n}^{1 / 2}\left(F_{1 n}\right)\right| \leq T_{n}^{1 / 2}\left(F_{n}-F_{1 n}+F_{0}\right) .
$$

If $m_{n}=\left[c_{0} k_{n}\right]$ and $c>2 c_{0}$, then, by Lemma A.9, we have

$$
n^{-1} T_{n}\left(F_{n}-F_{1 n}+F_{0}\right) \leq c_{0} c^{-1}\left\|f_{n}-f_{1 n}\right\|^{2} .
$$

Since the choice of $c$ is arbitrary, by Theorem 6.3, (A.45) and (A.46) imply (4.7) and (4.8).

Proof of $i$. in version of Theorem 4.4 and versions of Theorems 4.5, 4.8, 4.9, 4.10 follows from Theorem 6.3 and versions of Theorems 4.1 and 4.2 using the same reasoning as in subsection A.2. Proof of Theorem 6.2 is akin to proof of Theorem 4.7 and is omitted.

\section{A.5. Proof of Theorems of section 7 and Theorem 8.3}

Lemma A.10 given below allows to carry over corresponding reasoning for Brownian bridge $b(t), t \in(0,1)$, instead of empirical distribution functions.

Lemma A.10. For any $x>0$, we have

$$
P_{F_{n}}\left(n T^{2}\left(\hat{F}_{n}-F_{0}\right)<x\right)-P\left(T^{2}\left(b(t)+\sqrt{n}\left(F_{n}(t)-F_{0}(t)\right)\right)<x\right)=o(1)
$$

uniformly onto sequences c.d.f.'s $F_{n}$ such that $T\left(F_{n}-F_{0}\right)<c n^{-1 / 2}$.

If $\sqrt{n}\left(F_{n}-F_{0}\right) \rightarrow G$ in Kolmogorov - Smirnov distance, (A.47) has been proved Chibisov [3] without any statements of uniform convergence.

Lemma A.10 follows from Lemmas A.11 and A.13 given below after implementation of Hungary construction (see Th. 3, Ch. 12, section 1, [28]).

Lemma A.11. For any $x>0$, we have

$$
\begin{aligned}
& \mathbf{P}\left(T^{2}\left(b\left(F_{n}(t)\right)+\sqrt{n}\left(F_{n}(t)-F_{0}(t)\right)\right)<x\right) \\
& -\mathbf{P}\left(T^{2}\left(b(t)+\sqrt{n}\left(F_{n}(t)-F_{0}(t)\right)\right)<x\right)=o(1)
\end{aligned}
$$

uniformly onto sequences of c.d.f.'s $F_{n}$ such that $T\left(F_{n}-F_{0}\right)<c n^{-1 / 2}$.

Lemma A.11 follows from Lemmas A.12 and A.13 given below.

Lemma A.12. There holds

$$
\mathbf{E}\left[\left|T^{2}\left(b\left(F_{n}(t)\right)\right)-T^{2}(b(t))\right|\right]<c T^{1 / 4}\left(F_{n}-F_{0}\right) .
$$


Proof. We have

$$
\begin{aligned}
& \mathbf{E}^{2}\left[\mid T^{2}\left(b\left(F_{n}(t)\right)-T^{2}(b(t)) \mid\right] \leq \mathbf{E}^{2}\left[\mid\left(T\left(b\left(F_{n}(t)\right)-T(b(t))\right)\left(T\left(b\left(F_{n}(t)\right)+T(b(t))\right) \mid\right]\right.\right.\right. \\
& \leq \mathbf{E}\left[\left(\left(T\left(b\left(F_{n}(t)\right)\right)-T(b(t))\right)^{2}\right] \mathbf{E}\left[\left(T\left(b\left(F_{n}(t)\right)\right)+T(b(t))\right)^{2}\right]\right. \\
& \leq C \mathbf{E}\left[\left(\left(T\left(b\left(F_{n}(t)\right)-T(b(t))\right)^{2}\right] \leq C \mathbf{E}\left[T^{2}\left(b\left(F_{n}(t)\right)-b(t)\right)\right)\right]\right. \\
& =C \int_{0}^{1}\left(F_{n}(t)-F_{n}^{2}(t)-2 \min \left(F_{n}(t), F_{0}(t)\right)+2 F_{n}(t) F_{0}(t)+F_{0}(t)-F_{0}^{2}(t) d t\right. \\
& =C \int_{0}^{1} F_{n}(t)+F_{0}(t)-2 \min \left(F_{n}(t), F_{0}(t)\right)-\left(F_{n}(t)-F_{0}(t)\right)^{2} d t \\
& =C \int_{0}^{1}\left|F_{n}(t)-F_{0}(t)\right|-\left(F_{n}(t)-F_{0}(t)\right)^{2} d t \\
& \leq C \int_{0}^{1}\left|F_{n}(t)-F_{0}(t)\right| d t \leq T^{1 / 2}\left(F_{n}-F_{0}\right) .
\end{aligned}
$$

Lemma A.13. Densities of c.d.f.'s $\mathbf{P}\left(T^{2}\left(b(t)+n^{1 / 2}\left(F_{n}(t)-F_{0}(t)\right)\right) \leq x\right)$ are uniformly bounded onto the set of all c.d.f. $F_{n}$ such that $n T^{2}\left(F_{n}-F_{0}\right)<C$. Here $C$ is arbitrary.

Proof. Brownian bridge $b(t)$ admits representation

$$
b(t)=\sum_{j=1}^{\infty} \frac{\xi_{j}}{\pi j} \psi_{j}(t)
$$

where $\psi_{j}(t)=\sqrt{2} \sin (\pi j t)$ and $\xi_{j}, 1 \leq j<\infty$, are i.i.d. Gaussian random variables, $\mathbf{E} \xi_{j}=0$ and $\mathbf{E} \xi_{j}^{2}=1$.

Therefore, if $F_{n}(t)=\sum_{j=1}^{\infty} \theta_{n j} \psi_{j}$, then

$$
T^{2}\left(b(t)+n^{1 / 2}\left(F_{n}(t)-F_{0}(t)\right)\right)=\sum_{j=1}^{\infty}\left(\frac{\xi_{j}}{\pi j}+n^{1 / 2} \theta_{n j}\right)^{2} .
$$

The right hand-side of (A.51) is a sum of independent random variables. Thus it suffices to show that, for any $C$, random variables

$$
\left(\xi_{1}+n^{1 / 2} \theta_{n 1}\right)^{2}+\left(\xi_{2} / 2+n^{1 / 2} \theta_{n 2}\right)^{2}
$$

have uniformly bounded densities onto $n^{1 / 2}\left|\theta_{n 1}\right| \leq C$ and $n^{1 / 2}\left|\theta_{n 2}\right| \leq C$.

Densities $\left(\xi_{1}+a\right)^{2}$ and $\left(\xi_{2}+b\right)^{2}$ have wellknown analytical form, and proof of uniform boundedness of densities of $\left(\xi_{1}+a\right)^{2}+\frac{1}{4}\left(\xi_{2}+b\right)^{2}$ with $|a| \leq C$ and $|b| \leq C$ is obtained by routine technique. We omit these standard estimates. 
For proof of Theorem 7.1 it suffices to prove $i i$. Hungary construction allows to reduce reasoning to proof of corresponding statement for Brownian bridge $b(t), t \in[0,1]$. Thus Theorem 7.1 follows from Theorem 8.3.

Proof of Theorem 8.3. Denote $\zeta=\left\{\zeta_{j}\right\}_{1}^{\infty}, \zeta_{j}=\sigma_{j} \xi_{j}$.

Suppose opposite that (8.6) does not valid. Then there is subsequence of vectors $\boldsymbol{\eta}_{n}=\left\{\eta_{n j}\right\}_{1}^{\infty} \in \Upsilon(a)$ such that we have

$$
\lim _{n \rightarrow \infty} \mathbf{P}\left(T\left(\boldsymbol{\eta}_{n}+\boldsymbol{\zeta}\right) \leq x_{\alpha}\right) \geq 1-\alpha .
$$

Denote $\theta_{n j}=\kappa_{j} \eta_{n j}, 1 \leq j<\infty$.

There are $\boldsymbol{\theta}=\left\{\theta_{j}\right\}_{1}^{\infty}$ and subsequence $n_{i} \rightarrow \infty$ such that $\theta_{n_{i} j} \rightarrow \theta_{j}$ as $i \rightarrow \infty$ for each $j, 1 \leq j<\infty$.

Therefore there are sequences $C_{k} \rightarrow \infty$ and $i_{k} \rightarrow \infty$ as $k \rightarrow \infty$, such that

$$
\lim _{k \rightarrow \infty} \frac{\sum_{j<C_{k}} \theta_{n_{i_{k} j} j}^{2}}{\sum_{j<C_{k}} \theta_{j}^{2}}=1
$$

and

$$
\lim _{k \rightarrow \infty} \sum_{j<C_{k}}\left(\theta_{n_{i_{k}} j}-\theta_{j}\right)^{2}=0
$$

We consider two cases.

$i$. There holds

$$
\lim _{k \rightarrow \infty} \sum_{j>C_{k}} \theta_{n_{i_{k}} j}^{2}=0
$$

ii. There holds

$$
\sum_{j>C_{k}} \theta_{n_{i_{k}} j}^{2}>c \text { for all } k>k_{0}
$$

If $i$. holds, we have

$$
\mathbf{E}\left(\sum_{j>C_{k}} \kappa_{j} \zeta_{j} \theta_{n_{i_{k}} j}\right)^{2}=\sum_{j>C_{k}} \sigma_{j}^{2} \theta_{n_{i_{k}} j}^{2}=o(1)
$$

By (A.54), we get

$$
\mathbf{E}\left(\sum_{j<C_{k}} \kappa_{j} \zeta_{j}\left(\theta_{n_{i_{k}} j}-\theta_{j}\right)\right)^{2}=\sum_{j<C_{k}} \kappa_{j}^{2} \sigma_{j}^{2}\left(\theta_{n_{i_{k}} j}-\eta_{j}\right)^{2}=o(1) .
$$


By (A.55) and (A.56), we get

$$
\begin{aligned}
& \mathbf{P}\left(\sum_{j=1}^{\infty}\left(\kappa_{j} \zeta_{j}+\theta_{n_{i_{k}} j}\right)^{2}<x_{\alpha}\right) \\
& =\mathbf{P}\left(\sum_{j<C_{k}}\left(\kappa_{j} \zeta_{j}+\theta_{n_{i_{k}} j}\right)^{2}+\sum_{j>C_{k}} \kappa_{j}^{2} \zeta_{j}^{2}<x_{\alpha}\left(1+o_{P}(1)\right)\right) \\
& =\mathbf{P}\left(\sum_{j<C_{k}}\left(\kappa_{j} \zeta_{j}+\theta_{j}\right)^{2}+\sum_{j>C_{k}} \kappa_{j}^{2} \zeta_{j}^{2}<x_{\alpha}\left(1+o_{P}(1)\right)\right) \\
& <\mathbf{P}\left(\sum_{j=1}^{\infty} \kappa_{j}^{2} \zeta_{j}^{2}<x_{\alpha}\right)(1+o(1)) .
\end{aligned}
$$

where the last inequality follows from Lemma A.14 given below.

Lemma A.14. Let $\boldsymbol{\theta}=\left\{\theta_{j}\right\}_{1}^{\infty}$ be such that $\sum_{j=1}^{\infty} \theta_{j}^{2}>c$. Then there holds

$$
\mathbf{P}\left(\sum_{j=1}^{\infty} \kappa_{j}^{2} \zeta_{j}^{2}<x_{\alpha}\right)>\mathbf{P}\left(\sum_{j=1}^{\infty}\left(\kappa_{j} \zeta_{j}+\theta_{j}\right)^{2}<x_{\alpha}\right) .
$$

Proof. For simplicity of notation the reasoning will be provided for $\theta_{1} \neq 0$. Implementing Anderson Theorem [1], we get

$$
\begin{aligned}
& \mathbf{P}\left(\sum_{j=1}^{\infty}\left(\kappa_{j} \zeta_{j}+\theta_{j}\right)^{2}<x_{\alpha}\right) \\
& =(2 \pi)^{-1 / 2} \int_{-\kappa_{1}^{-1} \sigma_{1}^{-1} \sqrt{x_{\alpha}}-\eta_{1}}^{\kappa_{1}^{-1} \sigma_{1}^{-1} \sqrt{x_{\alpha}}-\eta_{1}} \exp \left\{-\frac{x^{2}}{2}\right\} \mathbf{P}\left(\sum_{j=2}^{\infty}\left(\kappa_{j} \zeta_{j}+\theta_{j}\right)^{2}<x_{\alpha}-\left(\kappa_{1} \sigma_{1} x+\theta_{1}\right)^{2}\right) d x \\
& \leq(2 \pi)^{-1 / 2} \int_{-\kappa_{1}^{-1} \sigma_{1}^{-1} \sqrt{x_{\alpha}}-\eta_{1}}^{\kappa_{1}^{-1} \sigma_{x_{\alpha}} \eta_{1}} \exp \left\{-\frac{x^{2}}{2}\right\} \mathbf{P}\left(\sum_{j=2}^{\infty} \kappa_{j}^{2} \zeta_{j}^{2}<x_{\alpha}-\left(\kappa_{1} \sigma_{1} x+\theta_{1}\right)^{2}\right) d x \\
& =\mathbf{P}\left(\left(\kappa_{1} \zeta_{1}+\theta_{1}\right)^{2}+\sum_{j=2}^{\infty} \kappa_{j}^{2} \zeta_{j}^{2}<x_{\alpha}\right)<\mathbf{P}\left(\sum_{j=1}^{\infty} \kappa_{j}^{2} \zeta_{j}^{2}<x_{\alpha}\right) .
\end{aligned}
$$

For the proof of last inequality in (A.58) it suffices to note that $\mathbf{P}\left(\kappa_{1} \zeta_{1}^{2}<x\right)>\mathbf{P}\left(\left(\kappa_{1} \zeta_{1}+\right.\right.$ $\left.\left.\theta_{1}\right)^{2}<x\right)$ for $x \in\left(0, x_{\alpha}\right)$, and, for any $\delta, 0<\delta<x_{\alpha}$, there is $\delta_{1}>0$ such that the function $\mathbf{P}\left(\kappa_{1} \zeta_{1}^{2}<x\right)-\mathbf{P}\left(\left(\kappa_{1} \zeta_{1}+\theta_{1}\right)^{2}<x\right)-\delta_{1}$ is positive onto interval $\left(\delta, x_{\alpha}\right)$.

Suppose $i$. holds. We suppose $n_{i_{k}}=n$. This allows to implement more simple notation. Then we have

$$
T\left(\boldsymbol{\eta}_{n}+\boldsymbol{\zeta}\right)==\sum_{j<C_{n}}\left(\kappa_{j} \zeta_{j}+\theta_{n j}\right)^{2}+J_{2 n},
$$


where

$$
\begin{aligned}
& J_{2 n}=\sum_{j \geq C_{n}} \kappa_{j}^{2} \zeta_{j}^{2}+2 \sum_{j \geq C_{n}} \kappa_{j} \zeta_{j} \theta_{n j} \\
& +\sum_{j \geq C_{n}} \theta_{n j}^{2}=J_{21 n}+2 J_{22 n}+J_{23 n} .
\end{aligned}
$$

We have

$$
J_{21 n}=o_{P}(1) \quad \text { and } \quad J_{22 n} \leq J_{21 n}^{1 / 2} J_{23 n}^{1 / 2}=o_{P}(1) .
$$

By (A.59) - (A.61), implementing Anderson Theorem [1], we get that, for any $0<\delta<c / 2$, there holds

$$
\begin{aligned}
& \mathbf{P}\left(\sum_{j=1}^{\infty}\left(\kappa_{j} \zeta_{j}+\theta_{n j}\right)^{2}<x\right) \leq \mathbf{P}\left(\sum_{j<C_{n}}\left(\kappa_{j} \zeta_{j}+\theta_{n j}\right)^{2} \leq x-c-o_{P}(1)\right) \\
& \leq \mathbf{P}\left(\sum_{j<C_{n}} \kappa_{j}^{2} \zeta_{j}^{2} \leq x-c+\delta\right)(1+o(1)) \\
& \leq \mathbf{P}\left(\sum_{j=1}^{\infty} \kappa_{j}^{2} \zeta_{j}^{2} \leq x-c+2 \delta\right)(1+o(1))<\mathbf{P}\left(\sum_{j=1}^{\infty} \kappa_{j}^{2} \zeta_{j}^{2} \leq x\right),
\end{aligned}
$$

where last inequality follows from Proposition 7.1 in [26].

Proof of version of Theorem 4.1. Let (4.3) hold. Then we have

$$
n \sum_{j=1}^{\infty} \frac{\theta_{n j}^{2}}{\pi^{2} j^{2}} \geq n \sum_{j<c_{2} k_{n}} \frac{\theta_{n j}^{2}}{\pi^{2} j^{2}} \geq c_{2}^{-2} n k_{n}^{-2} \sum_{j<c_{2} k_{n}} \theta_{n j}^{2} \asymp 1 .
$$

By (7.3), this implies sufficiency.

Proof of version of Theorem 4.2. Let (4.4) hold. Then we have

$$
\begin{aligned}
& n \sum_{j=1}^{\infty} \frac{\theta_{n j}^{2}}{\pi^{2} j^{2}}=n \sum_{j<c_{2} k_{n}} \frac{\theta_{n j}^{2}}{\pi^{2} j^{2}}+n \sum_{j>c_{2} k_{n}} \frac{\theta_{n j}^{2}}{\pi^{2} j^{2}} \\
& \leq o(1)+\left(c_{2} k_{n}\right)^{-2} n \sum_{j>c_{2} k_{n}} \theta_{n j}^{2} \asymp o(1)+\left(c_{2} k_{n}\right)^{-2} n^{1-2 r}=O\left(c_{2}^{-2}\right) .
\end{aligned}
$$

Since $c_{2}$ is arbitrary, then, by (7.3), (A.63) implies sufficiency.

Proof of Theorem 7.2. Proof of $i$ akin to proof of i. in Theorem 4.4. The statement follows from (4.3) and Lemma A.15 provided below.

Lemma A.15. Let $f_{n} \in \mathbb{B}_{2 \infty}^{s}\left(c_{1}\right)$ and $c n^{-r} \leq\left\|f_{n}\right\| \leq C n^{-r}$. Then, for $k_{n}=C_{1} n^{(1-2 r) / 2}(1+$ o(1)) with $C_{1}^{2 s}>2 c_{1} / c$, there holds

$$
\sum_{j=1}^{k_{n}} \theta_{n j}^{2}>\frac{c}{2} n^{-2 r}
$$


Proof of Lemma A.15 is akin to proof of Lemma A.3 and is omitted.

Reasoning in proof of $i$. is akin to proof of ii. in Theorem 4.4. Suppose opposite. Then there are $f=\sum_{j=1}^{\infty} \tau_{j} \phi_{j} \notin \mathbb{B}_{2 \infty}^{s}$ and a sequence $m_{l}, m_{l} \rightarrow \infty$ as $l \rightarrow \infty$, such that (A.9) holds. Define sequences $\boldsymbol{\eta}_{l}, n_{l}$ and $\tilde{f}_{l}$ by the same way as in the proof of Theorem 4.4.

Then we have

$$
n_{l} \asymp C_{l}^{-1 /(2 r)} m_{l}^{s / r}=C_{l}^{-1 /(2 r)} m_{l}^{\frac{2}{1-2 r}} .
$$

Therefore we get

$$
m_{l} \asymp C_{l}^{(1-2 r) /(4 r)} n_{l}^{\frac{1-2 r}{2}}
$$

Hence we get

$$
n_{l} \sum_{j=1}^{\infty} \frac{\eta_{l j}^{2}}{j^{2}} \leq n_{l} m_{l}^{-2} \sum_{j=m_{l}}^{\infty} \eta_{l j}^{2} \asymp n_{l}^{1-2 r} m_{l}^{-2} \asymp C_{l}^{\frac{2 r-1}{2 r}}=o(1) .
$$

By Theorem 7.1, (A.64) implies inconsistency of sequence of alternatives $\tilde{f}_{l}$.

Proof of Theorem 7.4. By Lemma A.10, it suffices to prove that, for any $\varepsilon>0$, there is $n_{0}(\varepsilon)$ such that, for $n>n_{0}(\varepsilon)$, the following inequality holds

$$
\begin{aligned}
& \mid \mathbf{P}\left(T^{2}\left(b\left(F_{n}(t)+F_{1 n}(t)-F_{0}(t)\right)+\sqrt{n}\left(F_{n}(t)+F_{1 n}(t)-2 F_{0}(t)\right)\right)>x_{\alpha}\right) \\
& -\mathbf{P}\left(T^{2}\left(b\left(F_{n}(t)\right)+\sqrt{n}\left(F_{n}(t)-F_{0}(t)\right)\right)>x_{\alpha}\right) \mid<\varepsilon .
\end{aligned}
$$

Since $T$ is a norm, by Lemma A.13, proof of (A.65) is reduced to proof that, for any $\delta_{1}>0$, there hold

$$
\mathbf{P}\left(\left|T\left(b\left(F_{n}(t)+F_{1 n}(t)-F_{0}(t)\right)\right)-T\left(b\left(F_{n}(t)\right)\right)\right|>\delta_{1}\right)=o(1),
$$

and there is sequence $\delta_{n}, \delta_{n} \rightarrow 0$ as $n \rightarrow \infty$, such that there holds

$$
n^{1 / 2}\left|T\left(F_{n}(t)+F_{1 n}(t)-2 F_{0}(t)\right)-T\left(F_{n}(t)-F_{0}(t)\right)\right|<\delta_{n} .
$$

Note that

$$
\begin{aligned}
& \left|T\left(b\left(F_{n}(t)+F_{1 n}(t)-F_{0}(t)\right)\right)-T\left(b\left(F_{n}(t)\right)\right)\right| \\
& \left.\leq T\left(b\left(F_{n}(t)\right)+F_{1 n}(t)-F_{0}(t)\right)-b\left(F_{n}(t)\right)\right)
\end{aligned}
$$

and

$$
\left|T\left(F_{n}(t)+F_{1 n}(t)-2 F_{0}(t)\right)-T\left(F_{n}(t)-F_{0}(t)\right)\right| \leq T\left(F_{1 n}(t)-F_{0}(t)\right) .
$$

By Lemma A.11, we have

$$
\mathbf{E} T^{2}\left(b\left(F_{n}(t)+F_{1 n}(t)-F_{0}(t)\right)-b\left(F_{n}(t)\right)\right) \leq T^{1 / 4}\left(F_{1 n}-F_{0}\right)=o(1) .
$$

By (A.68) and (A.70), we get (A.66).

Since sequence of alternatives $f_{1 n}$ is inconsistent, we have

$$
n T^{2}\left(F_{1 n}(t)-F_{0}(t)\right)=o(1)
$$

as $n \rightarrow \infty$. By (A.69) and (A.71), we get (A.67). 
Theorem 7.1, G1 and B reduce proof of Theorem 7.3 to the analysis of sums $\sum_{c k_{n}<j<C k_{n}} \theta_{n j}^{2}$ with $C>c$. Such an analysis has been provided in details in subsection A.2 with another parameters $r$ and $s$. We omit proof of Theorem 7.3.

\section{References}

[1] Anderson, T. (1955) The integral of a symmetric unimodal function. Proc.Amer.Math.Soc. 6(1) 170-176.

[2] Autin, F., Clausel,M., Jean-Marc Freyermuth, J. and Marteau C. (2018). Maxiset point of view for signal detection in inverse problems. arxiv 1803.05875.

[3] Chibisov, D.M. (1965) An investigation of the asymptotic power of tests of fit. Theor.Prob. Appl. $10421-437$.

[4] Cohen, A., DeVore, R., Kerkyacharian, G. and Picard, D. (2001). Maximal spaces with given rate of convergence for thresholding algorithms, Appl. Comput. Harmon. Anal. 11167191

[5] Engl, H., Hanke, M. and Neubauer, A. (1996). Regularization of Inverse Problems. Kluwer Academic Publishers.

[6] Ermakov, M.S. (1990) Minimax detection of a signal in a Gaussian white noise. Theory Probab. Appl., 35 667-679.

[7] Ermakov, M.S. (1997). Asymptotic minimaxity of chi-squared tests. Theory Probab. Appl. 42 589-610.

[8] Ermakov, M.S. (2003). On asymptotic minimaxity of kernel-based tests. ESAIM Probab. Stat. 7 279-312

[9] Ermakov, M.S. (2006). Minimax detection of a signal in the heteroscedastic Gaussian white noise. J. Math. Sci. (NY), 137 4516-4524.

[10] Ermakov, M.S. (2017). On consistent hypothesis testing. J. Math. Sci. (NY), 225 751-769.

[11] Ermakov, M.S. (2018). On asymptotically minimax nonparametric detection of signal in Gaussian white noise. Zapiski Nauchnih Seminarov POMI RAS. 474 124-138 (in Russian), arxiv.org 1705.07408.

[12] Gine E. and Nickl R. (2015) Mathematical Foundation of Infinite-Dimensional Statistical Models. Cambridge University Press Cambridge

[13] Gretton A., Borgwardt K., Rasch M., Scholkopf B. and Smola A. A kernel twosample test. Journal of Machine Learning Research, 13(Mar):723773, 2012.

[14] Ibragimov,I.A. and Khasminskii, R.Z. (1977). On the estimation of infinitely dimensional parameter in Gaussian white noise. Dokl.AN USSR 236 1053-1055.

[15] Ingster, Yu.I. (1987). On comparison of the minimax properties of Kolmogorov, $\omega^{2}$ and $\chi^{2}$-tests. Theory. Probab. Appl. 32 346-350.

[16] Ingster,Yu.I. and Suslina,I.A. (2002). Nonparametric Goodness-of-fit Testing under Gaussian Models. Lecture Notes in Statistics 169 Springer: N.Y.

[17] Ingster,Yu. I., Sapatinas, T. and Suslina, I. A. (2012) Minimax signal detection in ill-posed inverse problems. - Ann. Statist., 4015241549. 
[18] Johnstone, I. M. (2015). Gaussian estimation. Sequence and wavelet models. Book Draft http://statweb.stanford.edu/ imj/

[19] Kerkyacharian, G. and Picard, D. (1993). Density estimation by kernel and wavelets methods: optimality of Besov spaces. Statist. Probab. Lett. 18327 - 336.

[20] Kerkyacharian, G. and Picard, D. (2002). Minimax or maxisets? Bernoulli 8, 219253.

[21] Laurent, B., Loubes, J. M., and Marteau, C. (2011). Testing inverse problems: a direct or an indirect problem? J. Statist. Plann. Inference 141 1849-1861.

[22] Le Cam, L. and Schwartz, L. (1960). A necessary and sufficient conditions for the existence of consistent estimates. Ann.Math.Statist. 31 140-150.

[23] Le Cam, L. (1973). Convergence of estimates under dimensionality restrictions. Ann.Statist. 1 38-53.

[24] Lehmann, E.L. and Romano, J.P. (2005). Testing Statistical Hypothesis. Springer Verlag, NY.

[25] Lepski, O.V. and Tsybakov, A.B.(2000). Asymptotically exact nonparametric hypothesis testing in sup-norm and at a fixed point. Probab. Theory Related Fields, 117:1, 1748.

[26] Lifshits, M. (2012) Lectures on Gaussian Processes. Springer. NY.

[27] Rivoirard, V. (2004). Maxisets for linear procedures. Statist. Probab. Lett. 67 267275

[28] Shorack, G.R. and Wellner, J.A. (1986) Empirical Processes with Application to Statistics. J.Wiley Sons NY

[29] Schwartz, L. (1965). On Bayes procedures. Z.Wahrsch.Verw. Gebiete 4 10-26.

[30] Tsybakov, A. (2009). Introduction to Nonparametric Estimation. Berlin: Springer.

[31] Ulyanov, P. L. (1964). On Haar series. Mathematical Sbornik. 63(105):2 356-391. In Russian. 